TRANSACTIONS OF THE

AMERICAN MATHEMATICAL SOCIETY

Volume 357, Number 12, Pages 4813-4837

S 0002-9947(05)04007-9

Article electronically published on June 29, 2005

\title{
COFINALITY OF THE NONSTATIONARY IDEAL
}

\author{
PIERRE MATET, ANDRZEJ ROSŁANOWSKI, AND SAHARON SHELAH
}

\begin{abstract}
We show that the reduced cofinality of the nonstationary ideal $\mathcal{N} S_{\kappa}$ on a regular uncountable cardinal $\kappa$ may be less than its cofinality, where the reduced cofinality of $\mathcal{N} S_{\kappa}$ is the least cardinality of any family $\mathcal{F}$ of nonstationary subsets of $\kappa$ such that every nonstationary subset of $\kappa$ can be covered by less than $\kappa$ many members of $\mathcal{F}$. For this we investigate connections of the various cofinalities of $\mathcal{N} S_{\kappa}$ with other cardinal characteristics of ${ }^{\kappa} \kappa$ and we also give a property of forcing notions (called manageability) which is preserved in $<\kappa$-support iterations and which implies that the forcing notion preserves non-meagerness of subsets of $\kappa_{\kappa}$ (and does not collapse cardinals nor changes cofinalities).
\end{abstract}

\section{INTRODUCTION}

Let $\kappa$ be a regular uncountable cardinal. For $C \subseteq \kappa$ and $\gamma \leq \kappa$, we say that $\gamma$ is $a$ limit point of $C$ if $\bigcup(C \cap \gamma)=\gamma>0$. $C$ is closed unbounded if $C$ is a cofinal subset of $\kappa$ containing all its limit points less than $\kappa$. A set $A \subseteq \kappa$ is nonstationary if $A$ is disjoint from some closed unbounded subset $C$ of $\kappa$. The nonstationary subsets of $\kappa$ form an ideal on $\kappa$ denoted by $\mathcal{N} S_{\kappa}$. The cofinality of this ideal, $\operatorname{cof}\left(\mathcal{N} S_{\kappa}\right)$, is the least cardinality of a family $\mathcal{F}$ of nonstationary subsets of $\kappa$ such that every nonstationary subset of $\kappa$ is contained in a member of $\mathcal{F}$. The reduced cofinality of $\mathcal{N} S_{\kappa}, \overline{\operatorname{cof}}\left(\mathcal{N} S_{\kappa}\right)$, is the least cardinality of a family $\mathcal{F} \subseteq \mathcal{N} S_{\kappa}$ such that every nonstationary subset of $\kappa$ can be covered by less than $\kappa$ many members of $\mathcal{F}$. This paper addresses the question of whether $\overline{\operatorname{cof}}\left(\mathcal{N} S_{\kappa}\right)=\operatorname{cof}\left(\mathcal{N} S_{\kappa}\right)$. Note that

$$
\kappa^{+} \leq \overline{\operatorname{cof}}\left(\mathcal{N} S_{\kappa}\right) \leq \operatorname{cof}\left(\mathcal{N} S_{\kappa}\right) \leq 2^{\kappa},
$$

so under GCH we have $\overline{\operatorname{cof}}\left(\mathcal{N} S_{\kappa}\right)=\operatorname{cof}\left(\mathcal{N} S_{\kappa}\right)$.

Let $\kappa_{2}$ be endowed with the $\kappa$-box product topology, 2 itself considered discrete. We say that a set $W \subseteq{ }^{\kappa} 2$ is $\kappa$-meager if there is a sequence $\left\langle U_{\alpha}: \alpha<\kappa\right\rangle$ of dense open subsets of $\kappa_{2}$ such that $W \cap \bigcap_{\alpha<\kappa} U_{\alpha}=\emptyset$. The covering number for the category of the space $\kappa_{2}$, denoted $\operatorname{cov}\left(\mathbf{M}_{\kappa, \kappa}\right)$, is the least cardinality of any collection $\mathcal{X}$ of

Received by the editors March 3, 2003.

2000 Mathematics Subject Classification. Primary 03E05, 03E35, 03E55.

Key words and phrases. Nonstationary ideal, cofinality.

The second author thanks the University Committee on Research of the University of Nebraska at Omaha for partial support. He also thanks his wife, Małgorzata Jankowiak-Rosłanowska, for supporting him when he was preparing the final version of this paper.

The research of the third author was partially supported by the Israel Science Foundation. Publication 799 . 
$\kappa$-meager subsets of $\kappa_{2}$ such that $\bigcup \mathcal{X}=\kappa_{2}$. It is not hard to verify that

$$
\operatorname{cov}\left(\mathbf{M}_{\kappa, \kappa}\right) \leq \operatorname{cof}\left(\mathcal{N} S_{\kappa}\right) \leq\left(\overline{\operatorname{cof}}\left(\mathcal{N} S_{\kappa}\right)\right)^{<\kappa} .
$$

It follows that if $\overline{\operatorname{cof}}\left(\mathcal{N} S_{\kappa}\right)<\operatorname{cov}\left(\mathbf{M}_{\kappa, \kappa}\right)$ and the Singular Cardinals Hypothesis holds true, then $\operatorname{cf}\left(\overline{\operatorname{cof}}\left(\mathcal{N} S_{\kappa}\right)\right)<\kappa$ and $\operatorname{cof}\left(\mathcal{N} S_{\kappa}\right)=\left(\overline{\operatorname{cof}}\left(\mathcal{N} S_{\kappa}\right)\right)^{+}$. We prove:

Theorem 0.1. Assume GCH. Then there is a $\kappa$-complete, $\kappa^{+}{ }_{-}$cc forcing notion $\mathbb{P}$ such that

$$
\Vdash_{\mathbb{P}} \text { “ } \overline{\operatorname{cof}}\left(\mathcal{N} S_{\kappa}\right)=\kappa^{+\omega} \quad \text { and } \operatorname{cof}\left(\mathcal{N} S_{\kappa}\right)=\kappa^{+(\omega+1)} " .
$$

What about the consistency of " $\overline{\operatorname{cof}}\left(\mathcal{N} S_{\kappa}\right)$ is regular and $\overline{\operatorname{cof}}\left(\mathcal{N} S_{\kappa}\right)<\operatorname{cof}\left(\mathcal{N} S_{\kappa}\right)$ "? We establish:

Theorem 0.2. It is consistent, relative to the existence of a cardinal $\nu$ such that $o(\nu)=\nu^{++}$, that $\overline{\operatorname{cof}}\left(\mathcal{N} S_{\omega_{1}}\right)=\aleph_{\omega+1}$ and $\operatorname{cov}\left(\mathbf{M}_{\aleph_{1}, \aleph_{1}}\right)=\aleph_{\omega+2}$.

The structure of the paper is as follows. In Section 1, for each infinite cardinal $\mu \leq \kappa$ we introduce the $<\mu$-cofinality $\operatorname{cof}^{<\mu}\left(\mathcal{N} S_{\kappa}\right)$ and the $<\mu$-dominating number $\mathfrak{d}_{\kappa}^{<\mu}$, and we show that these two numbers are equal. Section 2 is concerned with a variant of $\mathfrak{d}_{\kappa}^{<\mu}$ denoted by $\mathfrak{d}_{\kappa}^{\mathrm{cl},<\mu}$ (where cl stands for "club"). We establish that $\mathfrak{d}_{\kappa}^{<\mu}=\mathfrak{d}_{\kappa}^{\mathrm{cl},<\mu}$ if $\mu>\omega$.

$\mathcal{N} S_{\kappa}$ is the smallest normal ideal on $\kappa$. Section 3 deals with $\mathcal{N} S_{\kappa, \lambda}^{\kappa}$, the smallest $\kappa$-normal ideal on $\mathcal{P}_{\kappa}(\lambda)$. We compute $\operatorname{cof}^{<\mu}\left(\mathcal{N} S_{\kappa, \lambda}^{\kappa}\right)$ and give examples of situations when $\operatorname{cof}^{<\mu}\left(\mathcal{N} S_{\kappa, \lambda}^{\kappa}\right)<\operatorname{cof}\left(\mathcal{N} S_{\kappa, \lambda}^{\kappa}\right)$.

In the following section we present some basic facts regarding the ideal of $\kappa$ meager subsets of $\kappa_{2}$ and its covering number.

The final three sections of the paper present the consistency results mentioned in Theorems 0.1, 0.2 above. First, in Section 5 we introduce manageability, a property of $<\kappa$-complete $\kappa^{+}$-cc forcing notions which implies preservation of non-meagerness of subsets of $\kappa_{\kappa}$ and which can be iterated. Next, in Section 6 , we define one-step forcing and verify that it has all required properties. The final section gives the applications obtained by iterating this forcing notion.

Notation 0.3. Our notation is rather standard and compatible with that of classical textbooks (like Jech [6]). In forcing we keep the older (Cohen's) convention that $a$ stronger condition is the larger one. Some of our conventions are listed below.

(1) For a forcing notion $\mathbb{P}, \Gamma_{\mathbb{P}}$ stands for the canonical $\mathbb{P}$-name for the generic filter in $\mathbb{P}$. With this one exception, all $\mathbb{P}$-names for objects in the extension via $\mathbb{P}$ will be denoted with a dot above (e.g. $\dot{\tau}, \dot{X})$. The weakest element of $\mathbb{P}$ will be denoted by $\emptyset_{\mathbb{P}}$ (and we will always assume that there is one, and that there is no other condition equivalent to it). In iterations, if $\overline{\mathbb{Q}}=\left\langle\mathbb{P}_{\zeta}, \dot{\mathbb{Q}}_{\zeta}: \zeta\left\langle\zeta^{*}\right\rangle\right.$ and $p \in \lim (\overline{\mathbb{Q}})$, then we keep the convention that $p(\alpha)=\dot{\emptyset}_{\dot{\mathbb{Q}}_{\alpha}}$ for $\alpha \in \zeta^{*} \backslash \operatorname{Dom}(p)$.

(2) Ordinal numbers will be denoted by $\alpha, \beta, \gamma, \delta, \varepsilon, \zeta, \xi$ and also by $i, j$ (with possible sub- and superscripts).

Infinite cardinal numbers will be called $\theta, \iota, \mu, \nu, \tau$ (with possible sub- and superscripts); $\kappa$ is our fixed regular uncountable cardinal, $\lambda$ will denote a fixed cardinal $>\kappa$ (in Section 3 ).

(3) By $\chi$ we will denote a sufficiently large regular cardinal and by $\mathcal{H}(\chi)$ the family of all sets hereditarily of size less than $\chi$. Moreover, we fix a well ordering $<_{\chi}^{*}$ of $\mathcal{H}(\chi)$. 
(4) A bar above a letter denotes that the object considered is a sequence; usually $\bar{X}$ will be $\left\langle X_{i}: i\langle\zeta\rangle\right.$, where $\zeta$ denotes the length of $\bar{X}$. For a set $A$ and a cardinal $\mu$, the set of all sequences of members of $A$ of length $\mu$ (length $<\mu$, respectively), will be denoted by $\mu_{A}\left(<\mu_{A}\right.$, respectively).

$$
\text { 1. } \operatorname{cof}^{<\mu}\left(\mathcal{N} S_{\kappa}\right)
$$

Definition 1.1. (1) For a set $A$ and a cardinal $\mu, \mathcal{P}_{\mu}(A)=\{a \subseteq A:|a|<\mu\}$.

(2) Given two infinite cardinals $\mu \leq \tau, u(\mu, \tau)$ is the least cardinality of a collection $A \subseteq \mathcal{P}_{\mu}(\tau)$ such that $\mathcal{P}_{\mu}(\tau)=\bigcup_{a \in A} \mathcal{P}(a)$.

Definition 1.2. Let $S$ be an infinite set and $\mathcal{J}$ an ideal on $S$ (containing all singletons).

(1) $\operatorname{cof}(\mathcal{J})$ is the least cardinality of any $\mathcal{X} \subseteq \mathcal{J}$ such that for every $A \in \mathcal{J}$, there is $B \in \mathcal{X}$ with $A \subseteq B$.

(2) $\operatorname{add}(\mathcal{J})$ is the least cardinality of any $\mathcal{X} \subseteq \mathcal{J}$ such that $\bigcup \mathcal{X} \notin \mathcal{J}$.

(3) For an infinite cardinal $\mu \leq \operatorname{add}(\mathcal{J}), \operatorname{cof}^{<\mu}(\mathcal{J})$ is the least cardinality of a family $\mathcal{X} \subseteq \mathcal{J}$ such that for every $A \in \mathcal{J}$, there is $\mathcal{Y} \in \mathcal{P}_{\mu}(\mathcal{X})$ such that $A \subseteq \bigcup \mathcal{Y}$.

(4) We let $\overline{\operatorname{cof}}(\mathcal{J})=\operatorname{cof}^{<\operatorname{add}(\mathcal{J})}(\mathcal{J})$.

The following proposition collects some trivialities.

Proposition 1.3. Let $S$ be an infinite set and $\mathcal{J}$ an ideal on $S$ that contains all singletons. Then:

(i) $\operatorname{cof}^{<\omega}(\mathcal{J})=\operatorname{cof}(\mathcal{J})$.

(ii) If $\mu, \nu$ are two infinite cardinals with $\mu \leq \nu \leq \operatorname{add}(\mathcal{J})$, then $\operatorname{cof}^{<\nu}(\mathcal{J}) \leq \operatorname{cof}^{<\mu}(\mathcal{J})$.

(iii) $\operatorname{cof}(\mathcal{J}) \leq u\left(\mu, \operatorname{cof}^{<\mu}(\mathcal{J})\right)$ for every infinite cardinal $\mu \leq \operatorname{add}(\mathcal{J})$.

(iv) $\operatorname{add}(\mathcal{J}) \leq \overline{\operatorname{cof}}(\mathcal{J})$.

The following is well known (see, e.g., Matet, Péan and Shelah [10]).

Lemma 1.4. Let $\mu$ be a regular infinite cardinal. Then $u\left(\mu, \mu^{+n}\right)=\mu^{+n}$ for every $n<\omega$.

Proposition 1.5. Let $S$ be an infinite set and $\mathcal{J}$ an ideal on $S$ such that $(\operatorname{add}(\mathcal{J}))^{+\omega}$ $\leq \operatorname{cof}(\mathcal{J})$. Then $(\operatorname{add}(\mathcal{J}))^{+\omega} \leq \overline{\operatorname{cof}}(\mathcal{J})$.

Proof. Use Lemma 1.4

With these preliminaries out of the way, we can concentrate on ideals on $\kappa$. If there is a family of size $\kappa^{+\omega}$ of pairwise almost disjoint cofinal subsets of $\kappa$, then there is a $\kappa$-complete ideal $\mathcal{J}$ on $\kappa$ such that $\overline{\operatorname{cof}}(\mathcal{J})<\operatorname{cof}(\mathcal{J})$ (see Matet and Pawlikowski [9]).

Proposition 1.6. Suppose $\mathcal{J}$ is a normal ideal on $\kappa$ and $\kappa$ is a limit cardinal. Then $\overline{\operatorname{cof}}(\mathcal{J})=\operatorname{cof}^{<\mu}(\mathcal{J})$ for some infinite cardinal $\mu<\kappa$.

Proof. Assume that the conclusion fails. Fix $\mathcal{X} \subseteq \mathcal{J}$ such that $|\mathcal{X}|=\overline{\operatorname{cof}}(\mathcal{J})$ and

$$
\mathcal{J}=\bigcup\left\{\mathcal{P}(\bigcup X): X \in \mathcal{P}_{\kappa}(\mathcal{X})\right\}
$$


Set $\mathcal{Y}=\{A \cup \beta: A \in \mathcal{X} \& \beta \in \kappa\}$. Note that $|\mathcal{Y}|=\overline{\operatorname{cof}}(\mathcal{J})$. For each infinite cardinal $\mu<\kappa$ we may select a set $B_{\mu} \in \mathcal{J}$ so that $B_{\mu} \not \subseteq \bigcup Y$ for any $Y \in \mathcal{P}_{\mu}(\mathcal{Y})$. Now let $B$ be the set of all $\alpha<\kappa$ such that $\alpha \in B_{\mu}$ for some infinite cardinal $\mu<\alpha$. Since $B \in \mathcal{J}$ (by normality of $\mathcal{J}$ ), there must be $X \in \mathcal{P}_{\kappa}(\mathcal{X})$ such that $B \subseteq \bigcup X$. Let $\tau$ be any infinite cardinal such that $|X|<\tau<\kappa$. Then $B_{\tau} \subseteq \bigcup_{A \in X}(A \cup(\tau+1))$, which is a contradiction.

Arguing as in Proposition 1.6, we get:

Proposition 1.7. Suppose $\mathcal{J}$ is a $\kappa$-complete ideal on $\kappa$ and $\nu$ is an uncountable limit cardinal $<\kappa$. Then there is an infinite cardinal $\mu<\nu$ such that $\operatorname{cof}^{<\nu}(\mathcal{J})=$ $\operatorname{cof}^{<\mu}(\mathcal{J})$. Moreover, the least such $\mu$ is either $\omega$, or a successor cardinal.

The remainder of this section is concerned with $\operatorname{cof}^{<\mu}\left(\mathcal{N} S_{\kappa}\right)$. Let us recall the definition of the bounding number $\mathfrak{b}_{\kappa}$ :

Definition 1.8. The bounding number $\mathfrak{b}_{\kappa}$ is the least cardinality of any $\mathcal{F} \subseteq{ }^{\kappa} \kappa$ with the property that for every $g \in{ }^{\kappa} \kappa$, there is $f \in \mathcal{F}$ such that

$$
|\{\alpha<\kappa: g(\alpha) \leq f(\alpha)\}|=\kappa .
$$

The following is proved in Matet and Pawlikowski 9 .

Proposition 1.9. $\quad$ (i) $\overline{\operatorname{cof}}\left(\mathcal{N} S_{\kappa}\right) \geq \mathfrak{b}_{\kappa}$.

(ii) If $\overline{\operatorname{cof}}\left(\mathcal{N} S_{\kappa}\right)=\mathfrak{b}_{\kappa}$, then $\overline{\operatorname{cof}}\left(\mathcal{N} S_{\kappa}\right)=\operatorname{cof}\left(\mathcal{N} S_{\kappa}\right)$.

Proposition 1.10. Let $\mu$ be an infinite cardinal $\leq \kappa$. Then

$$
\text { either } \operatorname{cf}\left(\operatorname{cof}^{<\mu}\left(\mathcal{N} S_{\kappa}\right)\right)<\mu, \quad \text { or } \operatorname{cf}\left(\operatorname{cof}^{<\mu}\left(\mathcal{N} S_{\kappa}\right)\right) \geq \mathfrak{b}_{\kappa} .
$$

Proof. Suppose to the contrary that $\mu \leq \operatorname{cf}\left(\operatorname{cof}^{<\mu}\left(\mathcal{N} S_{\kappa}\right)\right)=\tau<\mathfrak{b}_{\kappa}$. For $\alpha<\tau$ select $\mathcal{X}_{\alpha} \subseteq \mathcal{N} S_{\kappa}$ so that

(i) $\left|\mathcal{X}_{\alpha}\right|<\operatorname{cof}^{<\mu}\left(\mathcal{N} S_{\kappa}\right)$,

(ii) $\mathcal{X}_{\beta} \subseteq \mathcal{X}_{\alpha}$ for $\beta<\alpha$,

(iii) $\mathcal{N} S_{\kappa}=\bigcup\left\{\mathcal{P}(\bigcup X): X \in \mathcal{P}_{\mu}\left(\bigcup_{\alpha<\tau} \mathcal{X}_{\alpha}\right)\right\}$.

For $\alpha<\tau$, set $\mathcal{Y}_{\alpha}=\left\{A \cup \beta: A \in \mathcal{X}_{\alpha} \& \beta \in \kappa\right\}$ and pick $B_{\alpha} \in \mathcal{N} S_{\kappa}$ so that $B_{\alpha} \nsubseteq \bigcup Y$ for any $Y \in \mathcal{P}_{\mu}\left(\mathcal{Y}_{\alpha}\right)$. By a result of Balcar and Simon (see [2, Theorem 5.25]), there is $B \in \mathcal{N} S_{\kappa}$ such that $\left|B_{\alpha} \backslash B\right|<\kappa$ for every $\alpha<\tau$. Select $X \in \mathcal{P}_{\mu}\left(\bigcup_{\alpha<\tau} \mathcal{X}_{\alpha}\right)$ so that $B \subseteq \bigcup X$. There is $\gamma<\tau$ such that $X \subseteq \mathcal{X}_{\gamma}$. Then $B_{\gamma} \subseteq \bigcup_{A \in X}(A \cup \beta)$ for some $\beta \in \kappa$, which is a contradiction.

Definition 1.11. Let $\tau \leq \kappa$. A family $\mathcal{F} \subseteq{ }^{\kappa} \kappa$ is called

- a dominating family if

$$
\left(\forall h \in{ }^{\kappa} \kappa\right)(\exists f \in \mathcal{F})(\forall j<\kappa)(h(j)<f(j)),
$$

- $a<\tau$-dominating family if

$$
\left(\forall h \in{ }^{\kappa} \kappa\right)\left(\exists F \in \mathcal{P}_{\tau}(\mathcal{F})\right)(\forall j<\kappa)(h(j)<\sup \{f(j): f \in F\}) .
$$

We define dominating numbers $\mathfrak{d}_{\kappa}, \mathfrak{d}_{\kappa}^{<\tau}$ by

$$
\begin{aligned}
& \mathfrak{d}_{\kappa}=\min \left\{|\mathcal{F}|: \mathcal{F} \subseteq{ }^{\kappa} \kappa \text { is a dominating family }\right\}, \\
& \mathfrak{d}_{\kappa}^{<\tau}=\min \left\{|\mathcal{F}|: \mathcal{F} \subseteq{ }^{<}{ }_{\kappa} \text { is a }<\tau \text {-dominating family }\right\} .
\end{aligned}
$$

We let $\overline{\mathfrak{d}}_{\kappa}=\mathfrak{d}_{\kappa}^{<\kappa}$ and for an infinite cardinal $\mu<\kappa$ we put $\mathfrak{d}_{\kappa}^{\mu}=\mathfrak{d}_{\kappa}^{<\mu^{+}}$. 
Note that $\mathfrak{d}_{\kappa}^{<\omega}=\mathfrak{d}_{\kappa}$. Landver [7] established that $\operatorname{cof}\left(\mathcal{N} S_{\kappa}\right)=\mathfrak{d}_{\kappa}$. His result can be generalized as follows.

Theorem 1.12. Let $\mu$ be an infinite cardinal $\leq \kappa$. Then $\operatorname{cof}^{<\mu}\left(\mathcal{N} S_{\kappa}\right)=\mathfrak{d}_{\kappa}^{<\mu}$.

Proof. Set $\tau=\operatorname{cof}^{<\mu}\left(\mathcal{N} S_{\kappa}\right)$. First we will argue that $\mathfrak{d}_{\kappa}^{<\mu} \leq \tau$. Select a family $\mathcal{C}$ of size $\tau$ of closed unbounded subsets of $\kappa$ so that for every closed unbounded subset $D$ of $\kappa$, there is $X \in \mathcal{P}_{\mu}(\mathcal{C}) \backslash\{\emptyset\}$ with $\bigcap X \subseteq D$. For $U \in \mathcal{P}_{\omega}(\mathcal{C}) \backslash\{\emptyset\}$ define $f_{U} \in \kappa_{\kappa}$ by $f_{U}(\alpha)=\min (\bigcap U \backslash(\alpha+1))$. Note that $f_{V}(\alpha) \leq f_{U}(\alpha)$ whenever $V \in \mathcal{P}(U) \backslash\{\emptyset\}$. Now given $g \in \kappa_{\kappa}$, let $D$ be the set of all limit ordinals $\delta<\kappa$ such that $g(\alpha)<\delta$ for every $\alpha<\delta$. Pick $X \in \mathcal{P}_{\mu}(\mathcal{C}) \backslash\{\emptyset\}$ so that $\bigcap X \subseteq D$. Define $h \in{ }^{\kappa} \kappa$ by

$$
h(\alpha)=\sup \left\{f_{U}(\alpha): U \in \mathcal{P}_{\omega}(X) \backslash\{\emptyset\}\right\} .
$$

We are going to show that $g<h$. Let $\alpha<\kappa$ and $C \in X$. First, suppose that there is $W \in \mathcal{P}_{\omega}(X) \backslash\{\emptyset\}$ such that $h(\alpha)=f_{W}(\alpha)$. Then $h(\alpha)=f_{W \cup\{C\}}(\alpha)$ and hence $h(\alpha) \in C$. Next suppose that $f_{U}(\alpha)<h(\alpha)$ for all $U \in \mathcal{P}_{\omega}(X) \backslash\{\emptyset\}$. Then $h(\alpha)$ is a limit ordinal. Set $\iota=\operatorname{cf}(h(\alpha))$ and pick an increasing sequence $\left\langle\gamma_{\beta}: \beta<\iota\right\rangle$ cofinal in $h(\alpha)$. For $\beta<\iota$, select $T_{\beta} \in \mathcal{P}_{\omega}(X) \backslash\{\emptyset\}$ with $\gamma_{\beta}<f_{T_{\beta}}(\alpha)$, and set $\delta_{\beta}=f_{T_{\beta} \cup\{C\}}(\alpha)$. Note that $\delta_{\beta} \in C$. Obviously, the sequence $\left\langle\delta_{\beta}: \beta<\iota\right\rangle$ is cofinal in $h(\alpha)$, and consequently $h(\alpha) \in C$. Thus for each $\alpha<\kappa, h(\alpha)$ belongs to $\bigcap X$ and therefore to $D$. Since clearly $h(\alpha)>\alpha$, it follows that $h(\alpha)>g(\alpha)$.

It remains to show that $\mathfrak{d}_{\kappa}^{<\mu} \geq \tau$. Let $\mathfrak{F}$ be the set of all strictly increasing functions from $\kappa$ to $\kappa$. Select $\mathcal{F} \subseteq \mathfrak{F}$ so that

(a) $|\mathcal{F}|=\mathfrak{d}_{\kappa}^{<\mu}$, and

(b) given $g \in{ }^{\kappa} \kappa$, there is $F_{g} \in \mathcal{P}_{\mu}(\mathcal{F})$ such that

$$
(\forall \alpha<\kappa)\left(g(\alpha)<\sup \left\{f(\alpha): f \in F_{g}\right\}\right) .
$$

For $f \in \mathfrak{F}$, let $C_{f}$ be the set of all limit ordinals $\alpha<\kappa$ such that $f(\beta)<\alpha$ for every $\beta<\alpha$. Easily

$$
\mathcal{N} S_{\kappa}=\left\{A \subseteq \kappa:(\exists g \in \mathfrak{F})\left(A \cap C_{g}=\emptyset\right)\right\}
$$

(see, e.g., [9]) and (as $\bigcap_{f \in F_{g}} C_{f} \subseteq C_{g}$ for every $g \in \mathfrak{F}$ ) it follows that $\tau \leq|\mathcal{F}|$.

It follows from Propositions 1.6 and 1.7 and Theorem 1.12 that to determine the value of $\operatorname{cof}^{<\mu}\left(\mathcal{N} S_{\kappa}\right)$ for every infinite cardinal $\mu \leq \kappa$, it suffices to compute $\mathfrak{d}_{\kappa}$ and $\mathfrak{d}_{\kappa}^{\tau}$ for every infinite cardinal $\tau<\kappa$.

$$
\text { 2. } \mathfrak{d}_{\kappa}^{\mathrm{cl},<\mu}
$$

It is straightforward to check that $\mathfrak{d}_{\kappa}^{<\mu}$ is the least cardinality of a family $\mathcal{F} \subseteq \kappa_{\kappa}$ such that

$$
\left(\forall g \in{ }^{\kappa} \kappa\right)\left(\exists F \in \mathcal{P}_{\mu}(\mathcal{F})\right)(|\{\alpha \in \kappa: g(\alpha) \geq \sup \{f(\alpha): f \in F\}\}|<\kappa) .
$$

In this section we discuss the variant that arises if we replace "has cardinality $<\kappa$ " by "is nonstationary".

Definition 2.1. (1) $\mathfrak{d}_{\kappa}^{\text {cl }}$ is the least cardinality of a family $\mathcal{F} \subseteq{ }^{\kappa} \kappa$ with the property that for every $g \in{ }^{\kappa} \kappa$, there is $f \in \mathcal{F}$ such that

$$
\{\alpha \in \kappa: g(\alpha) \geq f(\alpha)\} \in \mathcal{N} S_{\kappa} .
$$


(2) For an infinite cardinal $\mu \leq \kappa, \mathfrak{d}_{\kappa}^{\mathrm{cl},<\mu}$ is the least cardinality of a family $\mathcal{F} \subseteq{ }^{\kappa} \kappa$ with the property that for every $g \in{ }^{\kappa} \kappa$, there is $F \in \mathcal{P}_{\mu}(\mathcal{F})$ such that

$$
\{\alpha \in \kappa: g(\alpha) \geq \sup \{f(\alpha): f \in F\}\} \in \mathcal{N} S_{\kappa} .
$$

Note that $\mathfrak{d}_{\kappa}^{\mathrm{cl},<\omega}=\mathfrak{d}_{\kappa}^{\mathrm{cl}}$. It is simple to check that $\operatorname{cf}\left(\mathfrak{d}_{\kappa}^{\mathrm{cl}}\right) \geq \mathfrak{b}_{\kappa}$.

Theorem 2.2. For every uncountable cardinal $\mu \leq \kappa$,

$$
\mathfrak{d}_{\kappa}^{\mathrm{cl},<\mu}=\mathfrak{d}_{\kappa}^{<\mu}
$$

Theorem 2.2 easily follows from the next two lemmas.

Lemma 2.3. Let $\mu$ be an uncountable limit cardinal $\leq \kappa$. Then $\mathfrak{d}_{\kappa}^{\mathrm{cl},<\mu}=\mathfrak{d}_{\kappa}^{\mathrm{cl},<\tau}$ for some infinite cardinal $\tau<\mu$.

Proof. The proof is similar to that of Proposition 1.7. Suppose that the conclusion fails. Fix a family $\mathcal{F} \subseteq{ }^{\kappa} \kappa$ such that $|\mathcal{F}|=\mathfrak{d}_{\kappa}^{\text {cl, }<\mu}$ and

$$
\left(\forall g \in{ }^{\kappa} \kappa\right)\left(\exists F \in \mathcal{P}_{\mu}(\mathcal{F})\right)\left(\{\alpha \in \kappa: g(\alpha) \geq \sup \{f(\alpha): f \in F\}\} \in \mathcal{N} S_{\kappa}\right) .
$$

For each infinite cardinal $\tau<\mu$ we may select $g_{\tau} \in{ }^{\kappa} \kappa$ so that for every $F \in \mathcal{P}_{\tau}(\mathcal{F})$ we have

$$
\left\{\alpha \in \kappa: g_{\tau}(\alpha) \geq \sup \{f(\alpha): f \in F\}\right\} \notin \mathcal{N} S_{\kappa} .
$$

Define $g \in{ }^{\kappa} \kappa$ so that $g(\alpha) \geq g_{\tau}(\alpha)$ for every infinite cardinal $\tau<\mu$ such that $\tau<\alpha$. Now pick $F \in \mathcal{P}_{\mu}(\mathcal{F})$ such that

$$
\{\alpha \in \kappa: g(\alpha) \geq \sup \{f(\alpha): f \in F\}\} \in \mathcal{N} S_{\kappa} .
$$

Let $\tau$ be any infinite cardinal with $|F|<\tau<\mu$. Obviously, $F \in \mathcal{P}_{\tau}(\mathcal{F})$ and

$$
\left\{\alpha \in \kappa: g_{\tau}(\alpha) \geq \sup \{f(\alpha): f \in F\}\right\} \in \mathcal{N} S_{\kappa},
$$

a contradiction.

To establish the following lemma, we adapt the proof of Theorem 5 in Cummings and Shelah [3].

Lemma 2.4. Let $\mu$ be a regular uncountable cardinal $\leq \kappa$. Then $\mathfrak{d}_{\kappa}^{<\mu}=\mathfrak{d}_{\kappa}^{\mathrm{cl},<\mu}$.

Proof. Select a family $\mathcal{F} \subseteq \kappa_{\kappa}$ such that

(a) every member of $\mathcal{F}$ is increasing,

(b) $|\mathcal{F}|=\mathfrak{d}_{\kappa}^{\mathrm{cl},<\mu}$, and

(c) for each $g \in \kappa_{\kappa}$, there is $F \in \mathcal{P}_{\mu}(\mathcal{F})$ such that

$$
\{\alpha \in \kappa: g(\alpha) \geq \sup \{f(\alpha): f \in F\}\} \in \mathcal{N} S_{\kappa} .
$$

We claim that the family

$$
\mathcal{F}^{*} \stackrel{\text { def }}{=}\left\{f \in{ }^{\kappa} \kappa:(\exists \alpha, \beta<\kappa)(\exists g \in \mathcal{F})(f\lceil\beta \equiv \alpha \& f \uparrow[\beta, \kappa)=g\lceil[\beta, \kappa))\}\right.
$$

is $<\mu$-dominating. So let $g \in \kappa_{\kappa}$. Stipulate that $g_{-1}=g$. By induction on $n \in \omega$ choose a closed unbounded subset $C_{n}$ of $\kappa, g_{n}, h_{n} \in{ }^{\kappa} \kappa$ and $F_{n} \in \mathcal{P}_{\mu}(\mathcal{F})$ so that

(i) $C_{n+1} \subseteq C_{n}$,

(ii) $g_{n-1}(\alpha)<\sup \left\{f(\alpha): f \in F_{n}\right\}$ for all $\alpha \in C_{n}$,

(iii) $h_{n}(\beta)=\min \left(C_{n} \backslash(\beta+1)\right)$,

(iv) $g_{n}(\beta)=\sup \left(\operatorname{Rng}\left(g_{n-1} \uparrow\left(h_{n}(\beta)+1\right)\right)\right)$. 
Note that, by (iii) and (iv), $g(\beta) \leq g_{0}(\beta) \leq g_{1}(\beta) \leq \ldots$ for all $\beta \in \kappa$. Set $F=\bigcup_{n \in \omega} F_{n}$ and $\zeta=\sup \left\{\min \left(C_{n}\right): n \in \omega\right\}$. We are going to show that $g(\gamma)<$ $\sup \{f(\gamma): f \in F\}$ whenever $\zeta<\gamma<\kappa$. To this end suppose that $\zeta<\gamma<\kappa$. By (i), there are $m \in \omega$ and $\xi \in \kappa$ such that $\xi=\sup \left(\gamma \cap C_{n}\right)$ whenever $m \leq n<\omega$. By (iii), $h_{m}(\xi) \geq \gamma$ and so (by (iv)) $g(\gamma) \leq g_{m-1}(\gamma) \leq g_{m}(\xi)$. Since $\gamma>\zeta$ we also have $\gamma \cap C_{m+1} \neq \emptyset$. Hence $\xi \in C_{m+1}$ and consequently, by (ii),

$g(\gamma) \leq g_{m}(\xi)<\sup \left\{f(\xi): f \in F_{m+1}\right\} \leq \sup \left\{f(\gamma): f \in F_{m+1}\right\} \leq \sup \{f(\gamma): f \in F\}$.

Theorem 2.2 implies that $\mathfrak{d}_{\kappa}^{\omega} \leq \mathfrak{d}_{\kappa}^{\mathrm{cl}} \leq \mathfrak{d}_{\kappa}$. We mention that it was shown in Cummings and Shelah $\underline{3}$, that $\mathfrak{d}_{\kappa}^{\text {cl }}=\mathfrak{d}_{\kappa}$ if $\kappa>\beth_{\omega}$.

$$
\text { 3. } \operatorname{cof}^{<\mu}\left(\mathcal{N} S_{\kappa, \lambda}^{\kappa}\right)
$$

Throughout this section $\lambda$ denotes a fixed cardinal $>\kappa$. Our object of study will be the ideal $\mathcal{N} S_{\kappa, \lambda}^{\kappa}$, a $\mathcal{P}_{\kappa}(\lambda)$ version of $\mathcal{N} S_{\kappa}$.

Definition 3.1. For a regular uncountable cardinal $\nu$ and a cardinal $\tau \geq \nu, \mathcal{J}_{\nu, \tau}$ is the set of all $A \subseteq \mathcal{P}_{\nu}(\tau)$ such that for some $a \in \mathcal{P}_{\nu}(\tau)$ we have $\{b \in A: a \subseteq b\}=\emptyset$.

It is straightforward to check that $\mathcal{J}_{\nu, \tau}$ is a $\nu$-complete ideal on $\mathcal{P}_{\nu}(\tau)$.

Definition 3.2. (1) An ideal $\mathcal{J}$ of $\mathcal{P}_{\kappa}(\lambda)$ is $\kappa$-normal if given $A \in \mathcal{J}^{+}$and $f: A \longrightarrow \kappa$ such that $f(a) \in a \cap \kappa$ for all $a \in A$, there is $B \in \mathcal{J}^{+} \cap \mathcal{P}(A)$ such that $f$ is constant on $B$.

(2) The smallest $\kappa$-normal ideal on $\mathcal{P}_{\kappa}(\lambda)$ containing $\mathcal{J}_{\kappa, \lambda}$ is denoted by $\mathcal{N} S_{\kappa, \lambda}^{\kappa}$.

(3) For $f \in{ }^{\kappa}\left(\mathcal{P}_{\kappa}(\lambda)\right)$ we let

$$
C_{f} \stackrel{\text { def }}{=}\left\{a \in \mathcal{P}_{\kappa}(\lambda): a \cap \kappa \neq \emptyset \text { and } \bigcup_{\alpha \in a \cap \kappa} f(\alpha) \subseteq a\right\}
$$

The following lemma is due to Abe.

Lemma 3.3 (Abe [1]). Let $A \subseteq \mathcal{P}_{\kappa}(\lambda)$. Then

$$
A \in \mathcal{N} S_{\kappa, \lambda}^{\kappa} \quad \text { if and only if } \quad\left(\exists f \in{ }^{\kappa}\left(\mathcal{P}_{\kappa}(\lambda)\right)\right)\left(A \cap C_{f}=\emptyset\right) \text {. }
$$

Our purpose in this section is to compute the value of $\operatorname{cof}^{<\mu}\left(\mathcal{N} S_{\kappa, \lambda}^{\kappa}\right)$. We will need an analogue of $\mathfrak{d}_{\kappa}^{<\mu}$ defined in Definition 3.4(1) below.

Definition 3.4. Let $\mu \leq \kappa$ be an infinite cardinal.

(1) $\mathfrak{d}_{\kappa, \lambda}^{\kappa,<\mu}$ is the least cardinality of a family $\mathcal{X}$ of functions from $\kappa$ to $\mathcal{P}_{\kappa}(\lambda)$ with the property that

$$
\left(\forall g \in{ }^{\kappa}\left(\mathcal{P}_{\kappa}(\lambda)\right)\right)\left(\exists X \in \mathcal{P}_{\mu}(\mathcal{X})\right)(\forall \alpha \in \kappa)\left(g(\alpha) \subseteq \bigcup_{f \in X} f(\alpha)\right) .
$$

(2) $\operatorname{cov}\left(\lambda, \kappa^{+}, \kappa^{+}, \mu\right)$ is the least cardinality of a family $\mathcal{X} \subseteq \mathcal{P}_{\kappa^{+}}(\lambda)$ such that

$$
\left(\forall B \in \mathcal{P}_{\kappa^{+}}(\lambda)\right)\left(\exists X \in \mathcal{P}_{\mu}(\mathcal{X})\right)(B \subseteq \bigcup X) .
$$

Theorem 3.5. Let $\mu$ be an infinite cardinal $\leq \kappa$. Then

$$
\operatorname{cof}^{<\mu}\left(\mathcal{N} S_{\kappa, \lambda}^{\kappa}\right)=\mathfrak{d}_{\kappa, \lambda}^{\kappa,<\mu}=\max \left\{\mathfrak{d}_{\kappa}^{<\mu}, \operatorname{cov}\left(\lambda, \kappa^{+}, \kappa^{+}, \mu\right)\right\} .
$$

Theorem 3.5 is an immediate consequence of Lemmas 3.63 .9 below. 
Lemma 3.6. Let $\mu$ be an infinite cardinal $\leq \kappa$. Then

$$
\operatorname{cov}\left(\lambda, \kappa^{+}, \kappa^{+}, \mu\right) \leq \operatorname{cof}^{<\mu}\left(\mathcal{N} S_{\kappa, \lambda}^{\kappa}\right) .
$$

Proof. By 3.3 we may pick a family $\mathcal{X} \subseteq{ }^{\kappa}\left(\mathcal{P}_{\kappa}(\lambda)\right)$ with the property that $|\mathcal{X}|=$ $\operatorname{cof}^{<\mu}\left(\mathcal{N} S_{\kappa, \lambda}^{\kappa}\right)$ and for every function $g: \kappa \longrightarrow \mathcal{P}_{\kappa}(\lambda)$ there is $X \in \mathcal{P}_{\mu}(\mathcal{X})$ such that $\bigcap_{f \in X} C_{f} \subseteq C_{g}$. For $f \in \mathcal{X}$, let $B_{f}=\kappa \cup \bigcup_{\alpha<\kappa} f(\alpha) \in \mathcal{P}_{\kappa^{+}}(\lambda)$.

Suppose now that $B \in \mathcal{P}_{\kappa^{+}}(\lambda)$. Pick a function $g: \kappa \longrightarrow \mathcal{P}_{\kappa}(\lambda)$ such that $B \subseteq \bigcup_{\alpha<\kappa} g(\alpha)$. There is $X \in \mathcal{P}_{\mu}(\mathcal{X})$ such that $\bigcap_{f \in X} C_{f} \subseteq C_{g}$. We are going to show that $B \subseteq \bigcup_{f \in X} B_{f}$. To this end suppose $\alpha<\kappa$ and let us argue that $g(\alpha) \subseteq \bigcup_{f \in X} B_{f}$. For $n<\omega$ let $a_{n} \in \mathcal{P}_{\kappa}\left(\bigcup_{f \in X} B_{f}\right)$ be defined by

$$
a_{0}=\{\alpha\} \quad \text { and } \quad a_{n+1}=a_{n} \cup \bigcup_{f \in X} \bigcup_{\beta \in a_{n} \cap \kappa} f(\beta),
$$

and let $a=\bigcup_{n<\omega} a_{n}$. Then $\alpha \in a \in \bigcap_{f \in X} C_{f} \subseteq C_{g}$ and consequently $g(\alpha) \subseteq a \subseteq$ $\bigcup_{f \in X} B_{f}$

Lemma 3.7. Let $\mu$ be an infinite cardinal $\leq \kappa$. Then $\mathfrak{d}_{\kappa}^{<\mu} \leq \operatorname{cof}^{<\mu}\left(\mathcal{N} S_{\kappa, \lambda}^{\kappa}\right)$.

Proof. By Theorem 1.12 it suffices to establish that $\operatorname{cof}^{<\mu}\left(\mathcal{N} S_{\kappa}\right) \leq \operatorname{cof}^{<\mu}\left(\mathcal{N} S_{\kappa, \lambda}^{\kappa}\right)$. Let a family $\mathcal{X} \subseteq{ }^{\kappa}\left(\mathcal{P}_{\kappa}(\lambda)\right)$ be such that $|\mathcal{X}|=\operatorname{cof}^{<\mu}\left(\mathcal{N} S_{\kappa, \lambda}^{\kappa}\right)$ and

$$
\left(\forall B \in \mathcal{N} S_{\kappa, \lambda}^{\kappa}\right)\left(\exists X \in \mathcal{P}_{\mu}(\mathcal{X}) \backslash\{\emptyset\}\right)\left(B \cap \bigcap_{f \in X} C_{f}=\emptyset\right) .
$$

For $f \in \mathcal{X}$, let $Z_{f}$ be the set of all limit ordinals $\alpha<\kappa$ such that

$$
(\forall \beta<\alpha)(f(\beta) \cap \kappa \subseteq \alpha) .
$$

Plainly, $Z_{f}$ is a closed unbounded subset of $\kappa$. Now given a closed unbounded subset $T$ of $\kappa$, set $B_{T}=\left\{a \in \mathcal{P}_{\kappa}(\lambda): a \cap \kappa \notin T\right\}$. A simple argument (see, e.g., [10]) shows that $B_{T} \in \mathcal{N} S_{\kappa, \lambda}^{\kappa}$. Hence there is $X_{T} \in \mathcal{P}_{\mu}(\mathcal{X}) \backslash\{\emptyset\}$ such that $B_{T} \cap \bigcap_{f \in X_{T}} C_{f}=\emptyset$. We will show that $\bigcap_{f \in X_{T}} Z_{f} \subseteq T$. Thus let $\alpha \in \bigcap_{f \in X_{T}} Z_{f}$. Setting $a=\alpha \cup \bigcup_{f \in X_{T}} \bigcup_{\beta<\alpha} f(\beta)$, it is easy to see that $a \cap \kappa=\alpha$ and $a \in \bigcap_{f \in X_{T}} C_{f}$. It follows that $\alpha=a \cap \kappa \in T$.

Lemma 3.8. Let $\mu$ be an infinite cardinal $\leq \kappa$. Then $\operatorname{cof}^{<\mu}\left(\mathcal{N} S_{\kappa, \lambda}^{\kappa}\right) \leq \mathfrak{d}_{\kappa, \lambda}^{\kappa,<\mu}$.

Proof. The inequality easily follows from the following observation.

Suppose $h: \kappa \longrightarrow \mathcal{P}_{\kappa}(\lambda)$ and $X \in \mathcal{P}_{\mu}\left({ }^{\kappa}\left(\mathcal{P}_{\kappa}(\lambda)\right)\right)$ are such that

$$
(\forall \alpha<\kappa)\left(h(\alpha) \subseteq \bigcup_{f \in X} f(\alpha)\right) .
$$

Then $\bigcap_{f \in X} C_{f} \subseteq C_{h}$.

Lemma 3.9. Let $\mu$ be an infinite cardinal $\leq \kappa$. Then

$$
\mathfrak{d}_{\kappa, \lambda}^{\kappa,<\mu} \leq \max \left\{\mathfrak{d}_{\kappa}^{<\mu}, \operatorname{cov}\left(\lambda, \kappa^{+}, \kappa^{+}, \mu\right)\right\} .
$$


Proof. Fix $\mathcal{F} \subseteq{ }^{\kappa} \kappa$ so that $|\mathcal{F}|=\mathfrak{d}_{\kappa}^{<\mu}$ and

$$
\left(\forall h \in{ }^{\kappa} \kappa\right)\left(\exists F \in \mathcal{P}_{\mu}(\mathcal{F})\right)(\forall \alpha<\kappa)(h(\alpha) \leq \sup \{f(\alpha): f \in F\}) .
$$

Also, fix $\mathcal{X} \subseteq \mathcal{P}_{\kappa^{+}}(\lambda)$ such that $|\mathcal{X}|=\operatorname{cov}\left(\lambda, \kappa^{+}, \kappa^{+}, \mu\right)$ and

$$
\mathcal{P}_{\kappa^{+}}(\lambda)=\bigcup\left\{\mathcal{P}(\bigcup X): X \in \mathcal{P}_{\mu}(\mathcal{X})\right\} .
$$

For each $a \in \mathcal{X}$, select a mapping $\varphi_{a}: \kappa \stackrel{\text { onto }}{\longrightarrow} a$. Now for $f \in \mathcal{F}$ and $a \in \mathcal{X}$ define $g_{f, a}: \kappa \longrightarrow \mathcal{P}_{\kappa}(\lambda)$ by

$$
g_{f, a}(\alpha)=\left\{\varphi_{a}(\delta): \delta<f(\alpha)\right\} .
$$

Suppose now that $g: \kappa \longrightarrow \mathcal{P}_{\kappa}(\lambda)$. By the choice of $\mathcal{X}$, there is $X \in \mathcal{P}_{\mu}(\mathcal{X})$ such that $\bigcup_{\alpha<\kappa} g(\alpha) \subseteq \bigcup X$. Choose $h \in{ }^{\kappa} \kappa$ such that

$$
(\forall \alpha<\kappa)\left(g(\alpha) \subseteq \bigcup_{a \in X}\left\{\varphi_{a}(\xi): \xi<h(\alpha)\right\}\right) .
$$

Next pick $F \in \mathcal{P}_{\mu}(\mathcal{F})$ such that $(\forall \alpha<\kappa)(h(\alpha) \leq \sup \{f(\alpha): f \in F\})$. Then

$$
(\forall \alpha<\kappa)\left(g(\alpha) \subseteq \bigcup_{f \in F} \bigcup_{a \in X} g_{f, a}(\alpha)\right) .
$$

Another formula worth noting is:

$$
\operatorname{cof}^{<\mu}\left(\mathcal{N} S_{\kappa, \lambda}^{\kappa}\right)=\max \left\{\operatorname{cof}^{<\mu}\left(\mathcal{N} S_{\kappa}\right), \operatorname{cof}^{<\mu}\left(\mathcal{J}_{\kappa^{+}, \lambda}\right)\right\}
$$

This identity follows from Theorems 1.12 and 3.5 and the next proposition.

Proposition 3.10. Let $\mu$ be an infinite cardinal $\leq \kappa$. Then $\operatorname{cov}\left(\lambda, \kappa^{+}, \kappa^{+}, \mu\right)=$ $\operatorname{cof}^{<\mu}\left(\mathcal{J}_{\kappa^{+}, \lambda}\right)$.

Proof. The result easily follows from the following observation.

Suppose that $\mathcal{X} \subseteq \mathcal{P}_{\kappa^{+}}(\lambda)$ and $X \in \mathcal{P}_{\mu}(\mathcal{X}) \backslash\{\emptyset\}$. Then

$$
\bigcap_{a \in X}\left\{c \in \mathcal{P}_{\kappa^{+}}(\lambda): a \subseteq c\right\}=\left\{c \in \mathcal{P}_{\kappa^{+}}(\lambda): \bigcup X \subseteq c\right\},
$$

and therefore for each $b \in \mathcal{P}_{\kappa^{+}}(\lambda)$,

$$
b \subseteq \bigcup X \quad \text { if and only if } \bigcap_{a \in X}\left\{c \in \mathcal{P}_{\kappa^{+}}(\lambda): a \subseteq c\right\} \subseteq\left\{c \in \mathcal{P}_{\kappa^{+}}(\lambda): b \subseteq c\right\} .
$$

We next consider special cases when $\operatorname{cof}^{<\mu}\left(\mathcal{N} S_{\kappa, \lambda}^{\kappa}\right)<\operatorname{cof}\left(\mathcal{N} S_{\kappa, \lambda}^{\kappa}\right)$.

Lemma 3.11. Let $\mu$ be an infinite cardinal $\leq \kappa$. Then $\operatorname{cov}\left(\lambda, \kappa^{+}, \kappa^{+}, \mu\right) \geq \lambda$.

Proof. It is shown in Matet, Péan and Shelah [11] that $\overline{\operatorname{cof}}\left(\mathcal{J}_{\kappa^{+}, \lambda}\right) \geq \lambda$. Now observe that (by Proposition 3.10) $\operatorname{cov}\left(\lambda, \kappa^{+}, \kappa^{+}, \mu\right) \geq \overline{\operatorname{cof}}\left(\mathcal{J}_{\kappa^{+}, \lambda}\right)$.

Lemma 3.12. Suppose $\lambda$ is singular and $\mu$ is a cardinal such that $\operatorname{cf}(\lambda)<\mu \leq \kappa$. Then $\operatorname{cov}\left(\lambda, \kappa^{+}, \kappa^{+}, \mu\right) \leq \sup \left\{u\left(\kappa^{+}, \nu\right): \kappa<\nu<\lambda\right\}$.

Proof. Let $\left\langle\lambda_{\xi}: \xi<\operatorname{cf}(\lambda)\right\rangle$ be an increasing sequence of cardinals cofinal in $\lambda$. Then, for every $a \in \mathcal{P}_{\kappa^{+}}(\lambda), a=\bigcup_{\xi<\mathrm{cf}(\lambda)} a \cap \lambda_{\xi}$. The desired inequality follows. 
Proposition 3.13. Let $\mu$ be an uncountable cardinal $\leq \kappa$. Then

$$
\operatorname{cof}^{<\mu}\left(\mathcal{N} S_{\kappa, \kappa+\omega}^{\kappa}\right)=\max \left\{\mathfrak{d}_{\kappa}^{<\mu}, \kappa^{+\omega}\right\} .
$$

Proof. By Lemmas 1.4, 3.11 and 3.12 we have

$$
\operatorname{cov}\left(\kappa^{+\omega}, \kappa^{+}, \kappa^{+}, \mu\right)=\kappa^{+\omega}
$$

so the result follows from Theorem 3.5 .

Thus, if $\mathfrak{d}_{\kappa}^{<\omega_{1}} \leq \kappa^{+\omega}$, then

$$
\operatorname{cof}^{<\omega_{1}}\left(\mathcal{N} S_{\kappa, \kappa}^{\kappa}+\omega\right)<\operatorname{cof}\left(\mathcal{N} S_{\kappa, \kappa+\omega}^{\kappa}\right) .
$$

Lemma 3.14. Assume the Singular Cardinals Hypothesis. If $\lambda \geq 2^{\kappa}$, then

$$
u\left(\kappa^{+}, \lambda\right)= \begin{cases}\lambda^{+} & \text {if } \mathrm{cf}(\lambda) \leq \kappa, \\ \lambda & \text { otherwise }\end{cases}
$$

Proof. Plainly, $\lambda \leq u\left(\kappa^{+}, \lambda\right) \leq \lambda^{\kappa}$. It follows immediately that $u\left(\kappa^{+}, \lambda\right)=\lambda$ if $\operatorname{cf}(\lambda)>\kappa$. For the other case, use the well-known fact (see, e.g., [10]) that $\operatorname{cf}\left(u\left(\kappa^{+}, \lambda\right)\right) \geq \kappa^{+}$.

Proposition 3.15. Assume the Singular Cardinals Hypothesis. If $\lambda \geq 2^{\kappa}$ and $\aleph_{0} \leq \mu \leq \kappa$, then

$$
\operatorname{cof}^{<\mu}\left(\mathcal{N} S_{\kappa, \lambda}^{\kappa}\right)= \begin{cases}\lambda^{+} & \text {if } \mu \leq \operatorname{cf}(\lambda) \leq \kappa, \\ \lambda & \text { otherwise }\end{cases}
$$

Proof. By Lemma 3.11 $\operatorname{cov}\left(\lambda, \kappa^{+}, \kappa^{+}, \mu\right) \geq \lambda \geq \mathfrak{d}_{\kappa} \geq \mathfrak{d}_{\kappa}^{<\mu}$, so by Theorem 3.5,

$$
\operatorname{cof}^{<\mu}\left(\mathcal{N} S_{\kappa, \lambda}^{\kappa}\right)=\operatorname{cov}\left(\lambda, \kappa^{+}, \kappa^{+}, \mu\right) \text {. }
$$

CASE: $\quad \operatorname{cf}(\lambda)>\kappa$.

By Lemmas 3.11 and 3.14 we have $\lambda \leq \operatorname{cov}\left(\lambda, \kappa^{+}, \kappa^{+}, \mu\right) \leq u\left(\kappa^{+}, \lambda\right) \leq \lambda$, and hence $\operatorname{cov}\left(\lambda, \kappa^{+}, \kappa^{+}, \mu\right)=\lambda$.

CASE: $\mu \leq \operatorname{cf}(\lambda) \leq \kappa$.

By Lemma 3.14 we know that

$$
\operatorname{cov}\left(\lambda, \kappa^{+}, \kappa^{+}, \mu\right) \leq u\left(\kappa^{+}, \lambda\right) \leq \lambda^{+} \quad \text { and } \quad \lambda^{+} \leq u\left(\kappa^{+}, \lambda\right) \leq\left(\operatorname{cov}\left(\lambda, \kappa^{+}, \kappa^{+}, \mu\right)\right)^{<\mu} \text {. }
$$

Since $\lambda^{<\mu}=\lambda$, it follows that $\operatorname{cov}\left(\lambda, \kappa^{+}, \kappa^{+}, \mu\right)=\lambda^{+}$.

CASE: $\operatorname{cf}(\lambda)<\mu$.

By Lemmas 3.11 , 3.12 and 3.14 we have

$$
\lambda \leq \operatorname{cov}\left(\lambda, \kappa^{+}, \kappa^{+}, \mu\right) \leq \sup \left\{u\left(\kappa^{+}, \nu\right): \kappa<\nu<\lambda\right\} \leq \lambda,
$$

and consequently $\operatorname{cov}\left(\lambda, \kappa^{+}, \kappa^{+}, \mu\right)=\lambda$.

Thus, if the Singular Cardinals Hypothesis holds, $\mu \leq \kappa$ and $\lambda \geq 2^{\kappa}$, then

$$
\operatorname{cof}^{<\mu}\left(\mathcal{N} S_{\kappa, \lambda}^{\kappa}\right)<\operatorname{cof}\left(\mathcal{N} S_{\kappa, \lambda}^{\kappa}\right) \quad \text { if and only if } \quad \operatorname{cf}(\lambda)<\mu .
$$




$$
\text { 4. } \operatorname{cov}\left(\mathbf{M}_{\kappa, \kappa}\right)
$$

Let us recall some basic facts and definitions related to the combinatorics of the $\kappa$-meager ideal $\mathbf{M}_{\kappa, \kappa}$ on ${ }^{\kappa} 2$.

Definition 4.1. (1) The Baire number $n(X)$ of a topological space $X$ (also called the Novak number of $X$ ) is the least number of nowhere dense subsets of $X$ needed to cover $X$.

(2) For a topological space $X$ and a cardinal $\mu$, the $(<\mu)$-complete ideal of subsets of $X$ generated by nowhere dense subsets of $X$ is denoted by $\mathbf{M}_{<\mu}(X)$; $\mathbf{M}_{<\mu^{+}}(X)$ will be also denoted by $\mathbf{M}_{\mu}(X)$. The ideal $\mathbf{M}_{\mu}(X)$ is the ideal of $\mu$-meager subsets of $X$.

(3) The space ${ }^{\kappa} \kappa$ (respectively $\kappa_{2}$ ) is endowed with the topology obtained by taking as basic open sets $\emptyset$ and $O_{s}$ for $s \in<\kappa_{\kappa}$ (respectively $s \in<\kappa_{2}$ ), where $O_{s}=\left\{f \in \kappa^{\kappa} \kappa: s \subseteq f\right\}$ (respectively $O_{s}=\left\{f \in{ }^{\kappa} 2: s \subseteq f\right\}$ ).

(4) The ideals of $\kappa$-meager subsets of $\kappa_{\kappa},{ }_{2}$ are denoted by $\mathbf{M}_{\kappa, \kappa}^{\kappa}$ and $\mathbf{M}_{\kappa, \kappa}$, respectively.

Remark 4.2. (1) Clearly, for a topological space $X, n(X)$ is the least number of open dense subsets of $X$ with empty intersection. If $\mu \leq n(X)$, then $\mathbf{M}_{<\mu}(X)$ is a proper ideal (i.e., $X \notin \mathbf{M}_{<\mu}(X)$ ).

(2) Following the tradition of the set theory of the reals, we may consider the covering number $\operatorname{cov}\left(\mathbf{M}_{<\mu}(X)\right)$ of the ideal $\mathbf{M}_{<\mu}(X)$ :

$$
\operatorname{cov}\left(\mathbf{M}_{<\mu}(X)\right)=\min \left\{|\mathcal{A}|: \mathcal{A} \subseteq \mathbf{M}_{<\mu}(X) \& \bigcup \mathcal{A}=X\right\} .
$$

By the definition, $n(X)=\operatorname{cov}\left(\mathbf{M}_{<\aleph_{0}}(X)\right)$. But also for every $\mu<n(X)$ we have $\operatorname{cov}\left(\mathbf{M}_{<\mu}(X)\right)=n(X)$; also $\operatorname{cov}\left(\mathbf{M}_{<n(X)}(X)\right)=\operatorname{cf}(n(X))$.

(3) Plainly, $n\left({ }^{\kappa} \kappa\right)>\kappa$ and $n\left(\kappa_{2}\right)>\kappa$ (remember, $\kappa$ is assumed to be regular).

Lemma 4.3. Suppose that $X$ is a topological space, $\mu<n(X)$, and $Y_{\alpha}$ are open subsets of $X($ for $\alpha<\mu)$. Assume also that $Y=\bigcap_{\alpha<\mu} Y_{\alpha}$ is dense in $X$. Then, if $Y$ is equipped with the subspace topology, $n(Y)=n(X)$.

Proof. Let $U_{\beta}$ (for $\beta<n(X)$ ) be open dense subsets of $X$ such that $\bigcap_{\beta<n(X)} U_{\beta}=\emptyset$. Then $U_{\beta} \cap Y$ are open dense subsets of $Y$ (remember $Y$ is dense) and

$$
\bigcap_{\beta<n(X)}\left(U_{\beta} \cap Y\right)=\emptyset .
$$

This shows that $n(Y) \leq n(X)$.

Now, let $V_{\beta} \subseteq Y$ (for $\beta<n(Y)$ ) be open dense subsets of $Y$ such that $\bigcap_{\beta<n(Y)} V_{\beta}=$ $\emptyset$. Take open subsets $U_{\beta}$ of $X$ such that $V_{\beta}=U_{\beta} \cap Y$ - clearly $U_{\beta}$ 's are dense in $X$ (as $Y$ is also). Then $\emptyset=\bigcap_{\beta<n(Y)}\left(U_{\beta} \cap Y\right)=\bigcap_{\beta<n(Y)} U_{\beta} \cap \bigcap_{\alpha<\mu} Y_{\alpha}$, and hence $n(X) \leq n(Y)+\mu$ and therefore $n(X) \leq n(Y)$.

Proposition 4.4. $\operatorname{cov}\left(\mathbf{M}_{\kappa, \kappa}^{\kappa}\right)=n\left(\kappa_{\kappa}\right)=n\left({ }^{\kappa} 2\right)=\operatorname{cov}\left(\mathbf{M}_{\kappa, \kappa}\right)$.

Proof. For $s \in<\kappa_{2}$ and $\alpha<\kappa$ let $F(s, \alpha) \in<\kappa_{2}$ be such that $\operatorname{lh}(F(s, \alpha))=$ $\operatorname{lh}(s)+\alpha+1$ and

$$
F(s, \alpha)\lceil\operatorname{lh}(s)=s, \quad F(s, \alpha)\lceil[\operatorname{lh}(s), \operatorname{lh}(s)+\alpha) \equiv 1, \quad \text { and } \quad F(s, \alpha)(\operatorname{lh}(s)+\alpha)=0 .
$$


Now, let $\pi:<\kappa \kappa \longrightarrow<\kappa_{2}$ be such that

- $\pi(\langle\rangle)=\langle\rangle, \pi(s \curlyvee\langle\alpha\rangle)=F(\pi(s), \alpha)$ for $s \in<\kappa \kappa$, and

- if $\left\langle s_{\zeta}: \zeta<\xi\right\rangle \subseteq<\kappa \kappa$ is $\triangleleft$-increasing, $\xi<\kappa, s=\bigcup_{\zeta<\xi} s_{\zeta}$,

then $\pi(s)=\bigcup_{\zeta<\xi} \pi\left(s_{\zeta}\right)$.

Then $\pi$ induces a one-to-one mapping $\pi^{*}:{ }^{\kappa} \kappa \longrightarrow{ }^{\kappa} 2: \eta \mapsto \bigcup_{\zeta<\kappa} \pi(\eta\lceil\zeta)$. The range of $\pi^{*}$ is

$$
\operatorname{Rng}\left(\pi^{*}\right)=\left\{\rho \in \kappa_{2}:(\forall \alpha<\kappa)(\exists \beta<\kappa)(\alpha<\beta \& \rho(\beta)=0)\right\} .
$$

Plainly, $\operatorname{Rng}\left(\pi^{*}\right)$ is the intersection of $\kappa$ many open dense subsets of $\kappa_{2}$. Moreover, $\pi^{*}$ is a homeomorphism from ${ }^{\kappa} \kappa$ onto $\operatorname{Rng}\left(\pi^{*}\right)$. Therefore, using Lemma 4.3, we get $n\left(\kappa_{\kappa}\right)=n\left(\operatorname{Rng}\left(\pi^{*}\right)\right)=n\left({ }{ }_{2}\right)$. The rest should be clear (remember Remark $4.2(2,3))$.

Proposition 4.5. $\operatorname{cov}\left(\mathbf{M}_{\kappa, \kappa}\right) \leq \mathfrak{d}_{\kappa}$.

Definition 4.6. $\mathbb{C}_{\mu, \kappa}$ is the forcing notion for adding $\mu$ Cohen functions in ${ }^{\kappa} \kappa$ with $<\kappa$-support. Thus a condition in $\mathbb{C}_{\mu, \kappa}$ is a function $q$ such that

$$
\operatorname{Dom}(q) \subseteq \mu \times \kappa, \quad \operatorname{Rng}(q) \subseteq \kappa \quad \text { and } \quad|q|<\kappa .
$$

The order of $\mathbb{C}_{\mu, \kappa}$ is the inclusion.

Proposition 4.7. Assume $2^{<\kappa}=\kappa<\mu$. Then $\Vdash_{\mathbb{C}_{\mu, \kappa}} " \operatorname{cov}\left(\mathbf{M}_{\kappa, \kappa}\right) \geq \mu$ ".

\section{Manageable Forcing NOtions}

In this section we introduce a property of forcing notions which is crucial for the consistency results presented later: $(\theta, \mu, \kappa)$-manageability. This property has three ingredients: an iterable variant of $\kappa^{+}$-cc (see Definition [5.1),$\kappa$-completeness and a special property implying preservation of non-meagerness of subsets of ${ }^{\kappa} \kappa$ (see Proposition [5.9). Since later we will work with $<\kappa$-support iterations, we also prove a suitable preservation theorem (see Theorem 5.11).

Throughout the section we will assume that our fixed (uncountable) regular cardinal $\kappa$ satisfies $2^{<\kappa}=\kappa$ (so also $\kappa^{<\kappa}=\kappa$ ).

Definition 5.1 (See Shelah [15, Definition 1.1] and [16, Definition 7]). Let $\mathbb{P}$ be a forcing notion and $\varepsilon<\kappa$ a limit ordinal.

(1) We define a game $\partial_{\varepsilon, \kappa}^{c c}(\mathbb{P})$ of two players, Player I and Player II. A play lasts $\varepsilon$ steps, and at each stage $\alpha<\varepsilon$ of the play $\bar{q}^{\alpha}, \bar{p}^{\alpha}, \varphi^{\alpha}$ are chosen so that:

- $\bar{q}^{0}=\left\langle\emptyset_{\mathbb{P}}: i<\kappa^{+}\right\rangle, \varphi^{0}: \kappa^{+} \longrightarrow \kappa^{+}: i \mapsto 0$.

- If $\alpha>0$, then Player I picks $\bar{q}^{\alpha}, \varphi^{\alpha}$ such that

(i) $\bar{q}^{\alpha}=\left\langle q_{i}^{\alpha}: i<\kappa^{+}\right\rangle \subseteq \mathbb{P}$ satisfies

$$
(\forall \beta<\alpha)\left(\forall i<\kappa^{+}\right)\left(p_{i}^{\beta} \leq q_{i}^{\alpha}\right),
$$

(ii) $\varphi^{\alpha}: \kappa^{+} \longrightarrow \kappa^{+}$is regressive, i.e., $\left(\forall i<\kappa^{+}\right)\left(\varphi^{\alpha}(i)<1+i\right)$.

- Player II answers, choosing a sequence $\bar{p}^{\alpha}=\left\langle p_{i}^{\alpha}: i<\kappa^{+}\right\rangle \subseteq \mathbb{P}$ such that $\left(\forall i<\kappa^{+}\right)\left(q_{i}^{\alpha} \leq p_{i}^{\alpha}\right)$. 
If at some stage of the game Player I does not have any legal move, then he loses. If the game lasted $\varepsilon$ steps, Player I wins a play $\left\langle\bar{q}^{\alpha}, \bar{p}^{\alpha}, \varphi^{\alpha}: \alpha<\varepsilon\right\rangle$ if there is a club $C$ of $\kappa^{+}$such that for each distinct members $i, j$ of $C$ satisfying $\operatorname{cf}(i)=\operatorname{cf}(j)=\kappa$ and $(\forall \alpha<\varepsilon)\left(\varphi^{\alpha}(i)=\varphi^{\alpha}(j)\right)$, the set

$$
\left\{p_{i}^{\alpha}: \alpha<\varepsilon\right\} \cup\left\{p_{j}^{\alpha}: \alpha<\varepsilon\right\}
$$

has an upper bound in $\mathbb{P}$.

(2) The forcing notion $\mathbb{P}$ satisfies condition $(*)_{\kappa}^{\varepsilon}$ if Player I has a winning strategy in the game $\partial_{\varepsilon, \kappa}^{c c}(\mathbb{P})$.

Remark 5.2. Condition $(*)_{\kappa}^{\varepsilon}$ is a strong version of $\kappa^{+}-\mathrm{cc}$ (easily, if $\varepsilon<\kappa$ is limit, $\kappa^{\varepsilon}=\kappa$, and $\mathbb{P}$ satisfies $(*)_{\kappa}^{\varepsilon}$, then $\mathbb{P}$ satisfies $\left.\kappa^{+}-\mathrm{cc}\right)$. This condition was used in a number of papers, e.g., to obtain a series of consistency results on partition relations; see Shelah and Stanley [17, [18], Shelah [14], 15], [16]. Its primary use comes from the fact that it is preserved in $<\kappa$-support iterations.

Proposition 5.3 (See Shelah [15, Iteration Lemma 1.3] and [16, Theorem 35]). Let $\varepsilon<\kappa$ be a limit ordinal, $\kappa=\kappa^{<\kappa}$. Suppose that $\overline{\mathbb{Q}}=\left\langle\mathbb{P}_{\xi}, \dot{\mathbb{Q}}_{\xi}: \xi<\gamma\right\rangle$ is a $<\kappa$-support iteration such that for each $\xi<\gamma$,

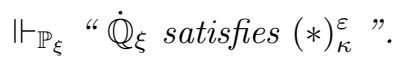

Then $\mathbb{P}_{\gamma}$ satisfies $(*)_{\kappa}^{\varepsilon}$.

Definition 5.4. A forcing notion $\mathbb{P}$ is $<\theta$-complete if every $\leq_{\mathbb{P}}$-increasing chain of length less than $\theta$ has an upper bound in $\mathbb{P}$. It is $\langle\theta-l u b-$ complete if every $\leq_{\mathbb{P}}$-increasing chain of length less than $\theta$ has a least upper bound in $\mathbb{P}$.

Definition 5.5. Let $\theta$ and $\mu$ be cardinals such that $\theta<\kappa$ and $\mu^{<\kappa}=\mu$. Let $\mathbb{P}$ be a $<\theta^{+}$-lub-complete forcing notion.

(1) A model $N \prec\left(\mathcal{H}(\chi), \in,<_{\chi}^{*}\right)$ is $(\mathbb{P}, \kappa, \mu)$-relevant if $\mathbb{P}, \mu \in N, \mu \subseteq N$, $|N|=\mu$ and $<\kappa N \subseteq N$.

(2) For a $(\mathbb{P}, \kappa, \mu)$-relevant model $N$ we define a game $\partial^{m}(N, \theta, \mathbb{P})$ of two players, He and She, as follows. A play lasts $\theta$ moves, and in the $i$ th move conditions $p_{i}, q_{i} \in \mathbb{P}$ are chosen so that:

- $q_{i} \in N \cap \mathbb{P}, q_{i} \leq p_{i}$,

- $(\forall j<i)\left(q_{j} \leq q_{i} \& p_{j} \leq p_{i}\right)$

- She chooses $p_{i}, q_{i}$ if $i$ is odd, He picks $p_{i}, q_{i}$ if $i$ is even.

She wins the play $\left\langle q_{i}, p_{i}: i<\theta\right\rangle$ whenever

if $p_{\theta}$ is a least upper bound of $\left\langle p_{i}: i<\theta\right\rangle$, and $q_{\theta}$ is a least upper bound of $\left\langle q_{i}: i<\theta\right\rangle$,

then $(\forall q \in N \cap \mathbb{P})\left(q_{\theta} \leq q \Rightarrow q, p_{\theta}\right.$ are compatible).

(3) The forcing notion $\mathbb{P}$ is weakly $(\theta, \mu, \kappa)$-manageable if (it is $<\theta^{+}$-lubcomplete and) there is an $x \in \mathcal{H}(\chi)$ (called a witness) such that for every $(\mathbb{P}, \kappa, \mu)$-relevant model $N \prec \mathcal{H}(\chi)$ with $x \in N$, She has a winning strategy in the game $\partial^{m}(N, \theta, \mathbb{P})$.

(4) The forcing notion $\mathbb{P}$ is $(\theta, \mu, \kappa)$-manageable if it is $<\kappa$-complete, weakly $(\theta, \mu, \kappa)$-manageable, and satisfies the condition $(*)_{\kappa}^{\theta}$.

Remark 5.6. Suppose that $\mathbb{P}$ is $<\theta^{+}$-lub-complete and $N$ is $(\mathbb{P}, \kappa, \mu)$-relevant. Then both players always have legal moves in the game $\partial^{m}(N, \theta, \mathbb{P})$. Moreover, if $\left\langle q_{i}, p_{i}\right.$ : $i<\theta\rangle$ is a (legal) play of $\partial^{m}(N, \theta, \mathbb{P})$, then there are least upper bounds $q_{\theta} \in N \cap \mathbb{P}$ of $\left\langle q_{i}: i<\theta\right\rangle$, and $p_{\theta} \in \mathbb{P}$ of $\left\langle p_{i}: i<\theta\right\rangle$ (and $q_{\theta} \leq p_{\theta}$ ). 
Definition 5.7. Let $N$ be a $(\mathbb{P}, \kappa, \mu)$-relevant model, and let $q \in N \cap \mathbb{P}, p \in \mathbb{P}$ be such that $q \leq p$. We say that a pair $\left(q^{*}, p^{*}\right)$ is an $N$-cover for $(q, p)$, if

- $q \leq q^{*} \in N \cap \mathbb{P}, p \leq p^{*} \in \mathbb{P}, q^{*} \leq p^{*}$, and

- every condition $q^{\prime} \in N \cap \mathbb{P}$ stronger than $q^{*}$ is compatible with $p^{*}$.

Lemma 5.8. Suppose that $\mathbb{P}$ is a $<\theta^{+}$-lub-complete forcing notion, $N$ is a $(\mathbb{P}, \kappa, \mu)$ relevant model, and She has a winning strategy in the game $\partial^{m}(N, \theta, \mathbb{P})$. Then:

(1) For all conditions $q \in N \cap \mathbb{P}$ and $p \in \mathbb{P}$ such that $q \leq p$, there is an $N$-cover $\left(q^{*}, p^{*}\right)$ for $(q, p)$.

(2) $N \cap \mathbb{P} \Leftarrow \mathbb{P}$.

Proof. (1) Consider a play $\left\langle q_{i}, p_{i}: i<\theta\right\rangle$ of $\partial^{m}(N, \theta, \mathbb{P})$ in which He starts with $q_{0}=q, p_{0}=p$, and then he always plays the $<_{\chi}^{*}$-first legal moves, and She uses her winning strategy. Let $q^{*} \in N \cap \mathbb{P}, p^{*} \in \mathbb{P}$ be least upper bounds of $\left\langle q_{i}: i<\theta\right\rangle$, $\left\langle p_{i}: i<\theta\right\rangle$, respectively. Plainly, as She won the play, the pair $\left(q^{*}, p^{*}\right)$ is an $N$-cover for $(q, p)$.

(2) Suppose that $\mathcal{A} \subseteq N \cap \mathbb{P}$ is a maximal antichain in $N \cap \mathbb{P}$, but $p \in \mathbb{P}$ is incompatible with all members of $\mathcal{A}$. Let $\left(q^{*}, p^{*}\right)$ be an $N$-cover for $\left(\emptyset_{\mathbb{P}}, p\right)$. The condition $q^{*}$ is compatible with some $q \in \mathcal{A}$, so let $q^{+} \in N \cap \mathbb{P}$ be such that $q^{+} \geq q^{*}$, $q^{+} \geq q \in \mathcal{A}$. By the choice of $\left(q^{*}, p^{*}\right)$ we know that the conditions $q^{+}$and $p^{*}$ are compatible, and hence $q$ and $p$ are compatible, a contradiction.

The rest follows from the elementarity of $N$.

Proposition 5.9. Assume $\theta<\kappa \leq \mu=\mu^{<\kappa}<\tau$. Suppose that a set $Y \subseteq{ }^{\kappa} \kappa$ cannot be covered by the union of less than $\tau$ nowhere dense subsets of $\kappa_{\kappa}$, and $\mathbb{P}$ is a weakly $(\theta, \mu, \kappa)$-manageable forcing notion not collapsing cardinals. Then

$$
\Vdash_{\mathbb{P}} \text { " } Y \text { is not the union of }<\tau \text { nowhere dense subsets of } \kappa_{\kappa} \text { ". }
$$

Proof. Let $\mathbb{P}$ be weakly $(\theta, \mu, \kappa)$-manageable with a witness $x \in \mathcal{H}(\chi)$. Suppose toward contradiction that a condition $q \in \mathbb{P}$ is such that

$$
q \Vdash \text { " } Y \text { is the union of }<\tau \text { nowhere dense subsets of } \kappa_{\kappa} \text { ". }
$$

Passing to a stronger condition if needed, we may assume that for some $\iota<\tau$ and $\mathbb{P}$-names $\dot{A}_{\xi}($ for $\xi<\iota)$ we have:

- $q \Vdash$ " $\dot{A}_{\xi} \subseteq<\kappa_{\kappa} \&\left(\forall s \in<\kappa_{\kappa}\right)\left(\exists t \in \dot{A}_{\xi}\right)(s \subseteq t)$ ", and

- $q \Vdash$ " $(\forall y \in Y)(\exists \xi<\iota)\left(\forall t \in \dot{A}_{\xi}\right)(t \nsubseteq \nsubseteq y)$ ”.

For each $\zeta<\iota$ pick a $(\mathbb{P}, \kappa, \mu)$-relevant model $N_{\zeta} \prec\left(\mathcal{H}(\chi), \in,<_{\chi}^{*}\right)$ such that $q,\left\langle\dot{A}_{\xi}\right.$ : $\xi<\iota\rangle, x, \zeta \in N_{\zeta}$. Then $\left|\bigcup_{\zeta<\iota} N_{\zeta}\right|=\iota \cdot \mu<\tau$, so we may pick a $y \in Y$ such that $y \in \mathcal{O}$ for all open dense subsets $\mathcal{O}$ of $\kappa_{\kappa}$ from $\bigcup_{\zeta<\iota} N_{\zeta}$. By our assumptions, there are $\xi<\iota$ and $p \geq q$ such that

$$
p \Vdash “\left(\forall t \in \dot{A}_{\xi}\right)(t \not \subseteq y) » .
$$

Let $\left(q^{*}, p^{*}\right)$ be an $N_{\xi}$-cover for $(q, p)$ (there is one by Lemma $\left.5.8(1)\right)$. Put

$$
A=\left\{s \in \kappa_{\kappa}^{<}:\left(\exists q^{\prime} \geq q^{*}\right)\left(q^{\prime} \Vdash s \in \dot{A}_{\xi}\right)\right\} .
$$

Clearly, $A \in N_{\xi}, A \subseteq N_{\xi}$, and $\mathcal{O}=\bigcup_{s \in A} O_{s} \in N_{\xi}$ is an open dense subset of $\kappa_{\kappa}$. Hence $s \subseteq y$ for some $s \in A$. Let $q^{\prime} \in N_{\xi} \cap \mathbb{P}$ be a condition stronger than $q^{*}$ and 
such that $q^{\prime} \Vdash s \in \dot{A}_{\xi}$. The condition $q^{\prime}$ is compatible with $p^{*}$, and so with $p$. Take a condition $q^{+}$stronger than both $q^{\prime}$ and $p$. Then

$$
q^{+} \Vdash " s \in \dot{A}_{\xi} \& s \subseteq y \text { \& } \quad \text { and } \quad q^{+} \Vdash "\left(\forall t \in \dot{A}_{\xi}\right)(t \nsubseteq y) ",
$$

a contradiction.

Corollary 5.10. Suppose that $\theta<\kappa \leq \mu=\mu^{<\kappa}$ and $\operatorname{cov}\left(\mathbf{M}_{\kappa, \kappa}\right)>\mu$. Let $\mathbb{P}$ be a $(\theta, \mu, \kappa)-$ manageable forcing notion. Then

$$
\Vdash_{\mathbb{P}} \cdots\left(\operatorname{cov}\left(\mathbf{M}_{\kappa, \kappa}\right)\right)^{\mathbf{V}} \leq \operatorname{cov}\left(\mathbf{M}_{\kappa, \kappa}\right) "
$$

Proof. Remembering Proposition 4.4, apply Proposition 5.9 to $\tau=\operatorname{cov}\left(\mathbf{M}_{\kappa, \kappa}\right)=$ $\operatorname{cov}\left(\mathbf{M}_{\kappa, \kappa}^{\kappa}\right)$ and $Y={ }_{\kappa} \kappa$ to get

$$
\Vdash_{\mathbb{P}} \text { " }\left(\kappa_{\kappa}\right) \mathbf{V} \text { is not the union of }<\tau \text { nowhere dense sets". }
$$

But this clearly implies $\Vdash_{\mathbb{P}} " \tau \leq \operatorname{cov}\left(\mathbf{M}_{\kappa, \kappa}\right)=\operatorname{cov}\left(\mathbf{M}_{\kappa, \kappa}^{\kappa}\right) "$.

Theorem 5.11. Assume that $\theta<\kappa \leq \mu=\mu^{<\kappa}$. Let $\overline{\mathbb{Q}}=\left\langle\mathbb{P}_{\xi}, \dot{\mathbb{Q}}_{\xi}: \xi<\gamma\right\rangle$ be $<\kappa$-support iteration such that for each $\xi<\gamma$,

$$
\Vdash_{\mathbb{P}_{\xi}} \text { “ } \dot{\mathbb{Q}}_{\xi} \text { is }(\theta, \mu, \kappa)-\text { manageable ". }
$$

Then $\mathbb{P}_{\gamma}$ is $(\theta, \mu, \kappa)$-manageable.

Proof. Let $\theta, \kappa, \mu$ and $\overline{\mathbb{Q}}$ be as in the assumptions of the theorem.

First note that the limits of $<\kappa$-support iterations of $<\kappa$-complete forcing notions satisfying the condition $(*)_{\kappa}^{\theta}$ are $<\kappa-$ complete $\kappa^{+}-$cc $\left(\right.$as $\kappa^{<\kappa}=\kappa$; remember Proposition 5.3). Therefore no such iteration collapses cardinals nor changes cofinalities nor adds sequences of ordinals of length $<\kappa$. Hence the assumed properties of $\theta, \kappa$ and $\mu$ hold in all intermediate extensions $\mathbf{V}^{\mathbb{P}} \xi$ and our assumption on $\dot{\mathbb{Q}}_{\xi}$ 's is meaningful.

Plainly, $\mathbb{P}_{\gamma}$ is $<\kappa$-complete, $<\theta^{+}$-lub-complete and satisfies condition $(*)_{\kappa}^{\theta}$. We have to show that $\mathbb{P}_{\gamma}$ is weakly $(\theta, \mu, \kappa)$-manageable.

For $\xi<\gamma$ let $\dot{x}_{\xi}$ be a $\mathbb{P}_{\xi}$-name for a witness for $\dot{\mathbb{Q}}_{\xi}$ being weakly manageable and let $\bar{x}=\left\langle\dot{x}_{\xi}: \xi<\gamma\right\rangle$. Suppose that $N \prec\left(\mathcal{H}(\chi), \in,<_{\chi}^{*}\right)$ is a $\left(\mathbb{P}_{\gamma}, \kappa, \mu\right)$-relevant model such that $(\bar{x}, \overline{\mathbb{Q}}) \in N$.

Since each $\mathbb{P}_{\xi}$ is $<\kappa$-complete and satisfies the $\kappa^{+}$-cc (and $\kappa+1 \subseteq N$ ), we know that if $\xi \in N \cap \gamma$ and $G_{\xi} \subseteq \mathbb{P}_{\xi}$ is generic over $\mathbf{V}$, then in $\mathbf{V}\left[G_{\xi}\right]$ we have

$N\left[G_{\xi}\right] \cap \mathbf{V}=N \quad$ and $\quad N\left[G_{\xi}\right] \prec\left(\mathcal{H}(\chi), \in,<_{\chi}^{*}\right) \mathbf{V}\left[G_{\xi}\right] \quad$ and $\quad<\kappa_{N}\left[G_{\xi}\right] \subseteq N\left[G_{\xi}\right]$.

Since clearly $\dot{\mathbb{Q}}_{\xi}^{G_{\xi}} \in N\left[G_{\xi}\right]$, we conclude that $N\left[G_{\xi}\right]$ is $\left(\dot{\mathbb{Q}}_{\xi}^{G_{\xi}}, \kappa, \mu\right)$-relevant, and $\dot{x}_{\xi}^{G_{\xi}} \in N\left[G_{\xi}\right]$. Therefore, She has a winning strategy in the game $\partial^{m}\left(N\left[G_{\xi}\right], \theta, \dot{\mathbb{Q}}_{\xi}^{G_{\xi}}\right)$. Let $\dot{s t}_{\xi}$ be a $\mathbb{P}_{\xi}$-name for such a strategy. We may assume that the strategy $\dot{s t}_{\xi}$ is such that

$(*)$ if $i<\theta$ is even and $q_{i}=p_{i}=\dot{\emptyset}_{\dot{\mathbb{Q}}_{\xi}}$,

then $\dot{s t}_{\xi}$ instructs Her to play $q_{i+1}=p_{i+1}=\dot{\emptyset}_{\dot{\mathbb{Q}}_{\xi}}$.

We define a strategy st for Her in the game $\partial^{m}\left(N, \theta, \mathbb{P}_{\gamma}\right)$ as follows. At an odd stage $i<\theta$ of the game, the strategy st first instructs Her to choose (side) conditions $q_{i}^{-}, p_{i}^{-} \in \mathbb{P}_{\gamma}$ and only then pick conditions $q_{i} \in N \cap \mathbb{P}_{\gamma}$ and $p_{i} \in \mathbb{P}_{\gamma}$ which are to be played. These conditions will be chosen so that if $\left\langle q_{j}, p_{j}: j<i\right\rangle$ is a legal play 
of $\partial^{m}\left(N, \theta, \mathbb{P}_{\gamma}\right)$ in which She uses st, and $q_{j}^{-}, p_{j}^{-}$are the side conditions picked by Her (for odd $j \leq i$ ), then

$(\alpha)_{i} \operatorname{Dom}\left(q_{i}\right)=\operatorname{Dom}\left(q_{i}^{-}\right)=\operatorname{Dom}\left(p_{i-1}\right) \cap N, \operatorname{Dom}\left(p_{i}^{-}\right)=\operatorname{Dom}\left(p_{i-1}\right)$,

$(\beta)_{i} p_{i-1} \leq p_{i}^{-} \leq p_{i}, q_{i-1} \leq q_{i}^{-} \leq p_{i}^{-}, q_{i-1} \leq q_{i} \leq p_{i}$,

$(\gamma)_{i}$ letting $\left(q_{j}^{*}, p_{j}^{*}\right)$ be $\left(q_{j}^{-}, p_{j}^{-}\right)$if $j \leq i$ is odd and $\left(q_{j}, p_{j}\right)$ if $j<i$ is even, for every $\xi \in \operatorname{Dom}\left(q_{i}\right)$ we have

$$
\begin{aligned}
p_{i}\left\lceil\xi \Vdash_{\mathbb{P}_{\xi}} \quad\right. & q_{i}(\xi)=q_{i}^{-}(\xi) \text { and } \\
& \text { the sequence }\left\langle q_{j}^{*}(\xi), p_{j}^{*}(\xi): j \leq i\right\rangle \text { is a legal play of } \\
& \partial^{m}\left(N\left[\Gamma_{\mathbb{P}_{\xi}}\right], \theta, \dot{\mathbb{Q}}_{\xi}\right) \text { in which She uses the strategy } \dot{s t}_{\xi} " .
\end{aligned}
$$

So suppose that $i<\theta$ is odd, $\left\langle q_{j}, p_{j}: j<i\right\rangle$ is a partial play of $\partial^{m}\left(N, \theta, \mathbb{P}_{\gamma}\right)$ in which She uses st (and the side conditions for odd $j<i$ are $q_{j}^{-}, p_{j}^{-}$), and the clauses $(\alpha)_{j}-(\gamma)_{j}$ hold for all odd $j<i$. Let $\left(q_{j}^{*}, p_{j}^{*}\right)$ be $\left(q_{j}^{-}, p_{j}^{-}\right)$if $j<i$ is odd and $\left(q_{j}, p_{j}\right)$ if $j<i$ is even.

We first declare that $\operatorname{Dom}\left(q_{i}^{-}\right)=\operatorname{Dom}\left(p_{i-1}\right) \cap N, \operatorname{Dom}\left(p_{i}^{-}\right)=\operatorname{Dom}\left(p_{i-1}\right)$ and $p_{i}^{-}(\zeta)=p_{i-1}(\zeta)$ for all $\zeta \in \operatorname{Dom}\left(p_{i}^{-}\right) \backslash N$. Next, by induction on $\xi \in \operatorname{Dom}\left(q_{i}^{-}\right)$ we define $q_{i}^{-}(\xi), p_{i}^{-}(\xi)$. So suppose that $\xi \in \operatorname{Dom}\left(q_{i}^{-}\right)$and $q_{i}^{-}\left\lceil\xi, p_{i}^{-}\lceil\xi\right.$ have been defined so that $q_{i-1} \mid \xi \leq q_{i}^{-}\left\lceil\xi \leq p_{i}^{-}\left\lceil\xi\right.\right.$ and $p_{i-1} \mid \xi \leq p_{i}^{-}\left\lceil\xi\right.$. Then, by clauses $(\gamma)_{j}$,

$$
\begin{aligned}
p_{i}^{-} \nmid \xi \Vdash_{\mathbb{P}_{\xi}} \quad \text { "the sequence }\left\langle q_{j}^{*}(\xi), p_{j}^{*}(\xi): j<i\right\rangle \text { is a legal play of } \\
\\
\partial^{m}\left(N\left[\Gamma_{\mathbb{P}_{\xi}}\right], \theta, \mathbb{Q}_{\xi}\right) \text { in which She uses the strategy } \operatorname{st}_{\xi} \text { ". }
\end{aligned}
$$

(Remember our assumption $(*)$ on $\dot{s t}_{\xi}$ and our convention regarding $\emptyset_{\mathbb{P}}$ stated in Notation $0.3(1)$.) Let $q_{i}^{-}(\xi)$ and $p_{i}^{-}(\xi)$ be $\mathbb{P}_{\xi}$-names for members of $\dot{\mathbb{Q}}_{\xi}$ such that

$$
q_{i}^{-}\left\lceil\xi \Vdash_{\mathbb{P}_{\xi}} " q_{i}^{-}(\xi) \in N\left[\Gamma_{\mathbb{P}_{\xi}}\right] \& q_{i-1}(\xi) \leq q_{i}^{-}(\xi) ",\right.
$$

and

$$
\begin{aligned}
p_{i}^{-} \mid \xi \Vdash_{\mathbb{P}_{\xi}} \quad & =\left(q_{i}^{-}(\xi), p_{i}^{-}(\xi)\right) \text { is what } \dot{s t}_{\xi} \text { tells Her to play } \\
& \text { as the answer to }\left\langle q_{j}^{*}(\xi), p_{j}^{*}(\xi): j<i\right\rangle " .
\end{aligned}
$$

(So $q_{i}^{-}(\xi)$ is a name for a member of $N\left[\Gamma_{\mathbb{P}_{\xi}}\right]$, but it does not have to be from $N$.) This completes the definition of $q_{i}^{-}, p_{i}^{-} \in \mathbb{P}_{\gamma}$. Now we use the fact that $\mathbb{P}_{\gamma}$ is $<\kappa$-complete and $\left|\operatorname{Dom}\left(q_{i}^{-}\right)\right|<\kappa$ to pick a condition $p_{i} \in \mathbb{P}_{\gamma}$ stronger than $p_{i}^{-}$ and names $\dot{\tau}_{\xi} \in N$ (for $\xi \in \operatorname{Dom}\left(q_{i}^{-}\right)$) such that $p_{i} \mid \xi \Vdash_{\mathbb{P}_{\xi}}$ " $q_{i}^{-}(\xi)=\dot{\tau}_{\xi}$ ". Since $<\kappa_{N} \subseteq N$, the sequence $\left\langle\dot{\tau}_{\xi}: \xi \in \operatorname{Dom}\left(q_{i}^{-}\right)\right\rangle$is in $N$. Hence we may find a condition $q_{i} \in N \cap \mathbb{P}_{\gamma}$ such that

- $\operatorname{Dom}\left(q_{i}\right)=\operatorname{Dom}\left(q_{i}^{-}\right)$, and

- for each $\xi \in \operatorname{Dom}\left(q_{i}\right)$,

$$
\Vdash_{\mathbb{P}_{\xi}} \text { " if } \dot{\tau}_{\xi} \geq q_{i-1}(\xi) \text {, then } q_{i}(\xi)=\dot{\tau}_{\xi} \text {, otherwise } q_{i}(\xi)=q_{i-1}(\xi) \text { ". }
$$

(For definiteness we pick the $<_{\chi}^{*}$-first $p_{i}, q_{i}$ as above.) It should be clear that $q_{i}^{-}, q_{i}, p_{i}^{-}, p_{i}$ satisfy conditions $(\alpha)_{i}-(\gamma)_{i}$. This finishes the description of the strategy st. Let us argue that it is a winning strategy for Her.

To this end suppose that $\left\langle q_{i}, p_{i}: i<\theta\right\rangle$ is the result of a play of $\partial^{m}\left(N, \theta, \mathbb{P}_{\gamma}\right)$ in which She uses st. Let $q_{\theta}, p_{\theta} \in \mathbb{P}_{\gamma}$ be least upper bounds of $\left\langle q_{i}: i<\theta\right\rangle,\left\langle p_{i}: i<\theta\right\rangle$, respectively. Then for every $\xi \in \operatorname{Dom}\left(p_{\theta}\right)$ we have

$$
p_{\theta}\left\lceil\xi \Vdash_{\mathbb{P}_{\xi}} \text { " } p_{\theta}(\xi) \text { is a least upper bound of }\left\langle p_{i}(\xi): i<\theta\right\rangle " .\right.
$$

We may also assume that $\operatorname{Dom}\left(q_{\theta}\right)=\bigcup_{i<\theta} \operatorname{Dom}\left(q_{i}\right)=\operatorname{Dom}\left(p_{\theta}\right) \cap N$. 
Let $q \in N \cap \mathbb{P}_{\gamma}$ be a condition stronger than $q_{\theta}$ (and thus stronger than all $q_{i}$ for $i<\theta)$. We define a condition $p \in \mathbb{P}_{\gamma}$ as follows. First, we declare that $\operatorname{Dom}(p)=$ $\operatorname{Dom}(q) \cup \operatorname{Dom}\left(p_{\theta}\right)$, and $p(\xi)=q(\xi)$ for $\xi \in \operatorname{Dom}(q) \backslash \operatorname{Dom}\left(p_{\theta}\right)$, and $p(\xi)=p_{\theta}(\xi)$ for $\xi \in \operatorname{Dom}\left(p_{\theta}\right) \backslash \operatorname{Dom}(q)=\operatorname{Dom}\left(p_{\theta}\right) \backslash N$. Now suppose that $\xi \in \operatorname{Dom}(q) \cap \operatorname{Dom}\left(p_{\theta}\right)$ and we have already defined $p\left\lceil\xi\right.$ so that $q \uparrow \xi \leq p \mid \xi$ and $p_{\theta} \uparrow \xi \leq p \mid \xi$. Then, by our choices,

$$
\begin{aligned}
p\left\lceil\xi \Vdash_{\mathbb{P}_{\xi}}\right. \text { " } & \text { the sequence }\left\langle q_{j}(\xi), p_{j}^{*}(\xi): j<\theta\right\rangle \text { is a legal play of } \\
& \partial^{m}\left(N\left[\Gamma_{\mathbb{P}_{\xi}}\right], \theta, \mathbb{Q}_{\xi}\right) \text { in which She uses the strategy st } \\
& q(\xi) \in N\left[\Gamma_{\mathbb{P}_{\xi}}\right] \text { is stronger than all } q_{j}(\xi) \text { for } j<\theta " .
\end{aligned}
$$

(Above, $p_{j}^{*}$ are as in the definition of st: either $p_{j}$ or $p_{j}^{-}$, depending on the parity of $j$.) Consequently,

$$
p\left\lceil\xi \Vdash " q(\xi) \text { and } p_{\theta}(\xi)\right. \text { are compatible ", }
$$

so we may pick a $\mathbb{P}_{\xi}$-name $p(\xi)$ for a condition in $\dot{\mathbb{Q}}_{\xi}$ such that

$$
p\left\lceil\xi \Vdash " q(\xi) \leq p(\xi) \text { and } p_{\theta}(\xi) \leq p(\xi) " .\right.
$$

This completes the choice of $p \in \mathbb{P}_{\gamma}$. Plainly, $p$ is an upper bound of $q$ and $p_{\theta}$ showing that they are compatible.

\section{Remark 5.12. Note that}

if $\mathbb{P}$ is weakly $(\theta, \mu, \kappa)$-manageable, then it satisfies the $\mu^{+}-$cc.

Hence we may use a slight modification of the proof of Theorem 5.11 to show (by induction on $\gamma$ ) that

$$
\begin{aligned}
& \text { if } \theta<\kappa=\kappa^{<\kappa}, \overline{\mathbb{Q}}=\left\langle\mathbb{P}_{\xi}, \dot{\mathbb{Q}}_{\xi}: \xi<\gamma\right\rangle \text { is a }(<\kappa) \text {-support iteration } \\
& \text { of }<\kappa \text {-complete weakly }(\theta, \kappa, \kappa) \text {-manageable forcing notions, } \\
& \text { then } \mathbb{P}_{\gamma} \text { is weakly }(\theta, \kappa, \kappa) \text {-manageable and } \kappa \text {-complete (and thus } \\
& \text { also } \kappa^{+} \text {-cc). }
\end{aligned}
$$

\section{THE ONE-STEP FORCING}

In this section we introduce a forcing notion $\mathbb{Q}$ for adding a small family of functions in ${ }^{\kappa} \kappa$ which $\tau$-dominates ${ }{ }_{\kappa} \cap \mathbf{V}$. Iterating this type of forcing notions we will get models with $\mathfrak{d}_{\kappa}^{\tau}$ small. Our forcing is (of course) manageable for suitable parameters, and thus it preserves non-meagerness of subsets of $\kappa$. Throughout this section we assume the following.

\section{Context 6.1. (i) $\tau=\operatorname{cf}(\tau)<\operatorname{cf}(\kappa)=\kappa=2^{<\kappa}$,}

(ii) $\bar{\mu}=\left\langle\mu_{\alpha}: \alpha<\tau\right\rangle$ is an increasing sequence of regular cardinals, $\kappa \leq \mu_{0}$,

(iii) $\left|\prod_{\alpha<\tau} \mu_{\alpha}\right|=2^{\kappa}$ and $\pi: \prod_{\alpha<\tau} \mu_{\alpha} \longrightarrow \kappa_{\kappa}$ is a bijection.

We will write $\pi_{\eta}$ for $\pi(\eta)$. Also, for a set $u \subseteq \prod_{\alpha<\tau} \mu_{\alpha}$ we let

$$
T(u) \stackrel{\text { def }}{=}\{\eta\lceil\alpha: \alpha<\tau \& \eta \in u\} .
$$

Definition 6.2. (1) We define a forcing notion $\mathbb{Q}=\mathbb{Q}(\pi, \bar{\mu}, \kappa)$ as follows.

A condition in $\mathbb{Q}$ is a tuple $p=(i, u, \bar{f}, g)=\left(i^{p}, u^{p}, \bar{f}^{p}, g^{p}\right)$ such that

(a) $i<\kappa, u \in \mathcal{P}_{\kappa}\left(\prod_{\alpha<\tau} \mu_{\alpha}\right)$,

(b) $\bar{f}=\left\langle f_{\sigma}: \sigma \in T(u)\right\rangle$ and $f_{\sigma}: i \longrightarrow \kappa$ for $\sigma \in T(u)$, 
(c) $g: u \longrightarrow i+1$, and if $\eta \in u, g(\eta) \leq j<i$, then

$$
\pi_{\eta}(j)<\sup \left\{f_{\eta \mid \alpha}(j): \alpha<\tau\right\}
$$

The order of $\mathbb{Q}$ is such that for $p, q \in \mathbb{Q}$ we have

$p \leq q$ if and only if

$i^{p} \leq i^{q}, u^{p} \subseteq u^{q}, g^{p} \subseteq g^{q}$ and $f_{\sigma}^{p} \subseteq f_{\sigma}^{q}$ for $\sigma \in T\left(u^{p}\right)$.

(2) For a set $U \subseteq \prod_{\alpha<\tau} \mu_{\alpha}$ we let $\mathbb{Q} \mid U=\left\{p \in \mathbb{Q}: u^{p} \subseteq U\right\}$, and for a condition $q \in \mathbb{Q}$ we put

$$
q \uparrow U=\left(i^{q}, u^{q} \cap U, \bar{f}^{q} \uparrow T\left(u^{q} \cap U\right), g^{q} \uparrow\left(u^{q} \cap U\right)\right) .
$$

Proposition 6.3. $\quad$ (1) $\mathbb{Q}$ is a $\quad$ (n-lub-complete forcing notion of size $2^{\kappa}$.

(2) Let $U \subseteq \prod_{\alpha<\tau} \mu_{\alpha}$ be of size $\leq \kappa$. Then $|\mathbb{Q}| U \mid \leq \kappa$.

Proof. (1) Plainly, $(\mathbb{Q}, \leq)$ is a partial order of size $2^{\kappa}$. To prove the completeness suppose that $\left\langle p_{\xi}: \xi<\xi^{*}\right\rangle$ is an $\leq$-increasing sequence of members of $\mathbb{Q}$ and $\xi^{*}<\kappa$. Put

$$
i^{q}=\sup _{\xi<\xi^{*}} i^{p_{\xi}}, \quad u^{q}=\bigcup_{\xi<\xi^{*}} u^{p_{\xi}}, \quad g^{q}=\bigcup_{\xi<\xi^{*}} g^{p_{\xi}}
$$

and $f_{\sigma}^{q}=\bigcup\left\{f_{\sigma}^{p_{\xi}}: \xi<\xi^{*} \& \sigma \in T\left(u^{p_{\xi}}\right)\right\}$ for $\sigma \in T\left(u^{q}\right)$. Clearly, $q=\left(i^{q}, u^{q}, \bar{f}^{q}, g^{q}\right) \in$ $\mathbb{Q}$ is the least upper bound of $\left\langle p_{\xi}: \xi<\xi^{*}\right\rangle$.

(2) Should be clear.

Proposition 6.4. The forcing notion $\mathbb{Q}$ satisfies the condition $(*)_{\kappa}^{\varepsilon}($ see Definition 5.1(2)) for any limit ordinal $\varepsilon<\kappa$.

Proof. Let $\varepsilon<\kappa$ be a limit ordinal. To give the winning strategy for Player I in the game $\partial_{\varepsilon, \kappa}^{\mathrm{cc}}(\mathbb{Q})$ we need two technical observations.

Claim 6.4.1. If $p, q \in \mathbb{Q}$ are such that $i^{p}=i^{q}$ and $g^{p} \uparrow\left(u^{p} \cap u^{q}\right)=g^{q} \uparrow\left(u^{p} \cap u^{q}\right)$ and $f_{\sigma}^{p}=f_{\sigma}^{q}$ for $\sigma \in T\left(u^{p}\right) \cap T\left(u^{q}\right)$, then the conditions $p, q$ have a least upper bound.

Proof of the Claim. Let $i^{r}=i^{p}=i^{q}, u^{r}=u^{p} \cup u^{q}, g^{r}=g^{p} \cup g^{q}$ and

$$
f_{\sigma}^{r}= \begin{cases}f_{\sigma}^{p} & \text { if } \sigma \in T\left(u^{p}\right), \\ f_{\sigma}^{q} & \text { if } \sigma \in T\left(u^{q}\right) .\end{cases}
$$

Then $r=\left(i^{r}, u^{r}, \bar{f}^{r}, g^{r}\right) \in \mathbb{Q}$ is the least upper bound of $p, q$.

Claim 6.4.2. Suppose $\bar{q}=\left\langle q_{j}: j\left\langle\kappa^{+}\right\rangle \subseteq \mathbb{Q}\right.$. Then there is a regressive function $\varphi_{\bar{q}}: \kappa^{+} \longrightarrow \kappa^{+}$such that

if $j<j^{\prime}<\kappa^{+}, \operatorname{cf}(j)=\operatorname{cf}\left(j^{\prime}\right)=\kappa$ and $\varphi_{\bar{q}}(j)=\varphi_{\bar{q}}\left(j^{\prime}\right)$,

then $i^{q_{j}}=i^{q_{j^{\prime}}}$, and $g^{q_{j}} \uparrow\left(u^{q_{j}} \cap u^{q_{j^{\prime}}}\right)=g^{q_{j^{\prime}}} \uparrow\left(u^{q_{j}} \cap u^{q_{j^{\prime}}}\right)$, and $f_{\sigma}^{q_{j}}=f_{\sigma}^{q_{j^{\prime}}}$ for $\sigma \in T\left(u^{q_{j}}\right) \cap T\left(u^{q_{j^{\prime}}}\right)$.

Proof of the Claim. Take a sequence $\left\langle\eta_{\xi}: \xi<\kappa^{+}\right\rangle \subseteq \prod_{\alpha<\tau} \mu_{\alpha}$ such that for each $j<$ $\kappa^{+}$of cofinality $\kappa$ and an $\alpha<j$ we have $u^{q_{\alpha}} \subseteq\left\{\eta_{\xi}: \xi<j\right\}$. Let $U=\left\{\eta_{\xi}: \xi<\kappa^{+}\right\}$ and $U_{j}=\left\{\eta_{\xi}: \xi<j\right\}$ for $j<\kappa^{+}$. By Proposition [6.3(2) we know that $|\mathbb{Q}| U_{j} \mid \leq \kappa$ (for $j<\kappa^{+}$) and $|\mathbb{Q}| U \mid \leq \kappa^{+}$, and hence we may pick a mapping $\psi_{0}: \kappa^{+} \longrightarrow \mathbb{Q} \mid U$ such that

$$
\left(\forall j<\kappa^{+}\right)\left(\operatorname{cf}(j)=\kappa \Rightarrow \operatorname{Rng}\left(\psi_{0}\lceil j)=\mathbb{Q}\left\lceil U_{j}\right) .\right.\right.
$$

Also, for $j<\kappa^{+}$, let $F\left(U_{j}\right)$ be the set

$$
\left\{\bar{f}=\left\langle f_{\sigma}: \sigma \in \operatorname{Dom}(\bar{f})\right\rangle: \operatorname{Dom}(\bar{f}) \in \mathcal{P}_{\kappa}\left(T\left(U_{j}\right)\right) \&(\forall \sigma \in \operatorname{Dom}(\bar{f}))\left(f_{\sigma} \in{ }^{<\kappa} \kappa\right)\right\},
$$


and $F(U)=\bigcup_{j<\kappa^{+}} F\left(U_{j}\right)$. Note that $\left|F\left(U_{j}\right)\right| \leq \kappa$ and $|F(U)| \leq \kappa^{+}$. Choose a function $\psi_{1}: \kappa^{+} \longrightarrow F(U)$ such that

$$
\left(\forall j<\kappa^{+}\right)\left(\operatorname{cf}(j)=\kappa \Rightarrow \operatorname{Rng}\left(\psi_{1}\lceil j)=F\left(U_{j}\right)\right) .\right.
$$

Finally, let $c: \kappa^{+} \times \kappa^{+} \longrightarrow \kappa^{+}$be a bijection such that

$$
\left(\forall j<\kappa^{+}\right)(\operatorname{cf}(j)=\kappa \Rightarrow \operatorname{Rng}(c \uparrow(j \times j))=j) .
$$

Now let $\varphi_{\bar{q}}: \kappa^{+} \longrightarrow \kappa^{+}$be a regressive function such that for $j<\kappa^{+}$of cofinality $\kappa$ we have

$$
\varphi_{\bar{q}}(j)=c\left(\min \left\{\alpha<\kappa^{+}: \psi_{0}(\alpha)=q_{j}\left\lceil U_{j}\right\}, \min \left\{\alpha<\kappa^{+}: \psi_{1}(\alpha)=\bar{f}^{q_{j}} \uparrow T\left(U_{j}\right)\right\}\right) .\right.
$$

Easily, $\varphi_{\bar{q}}$ is as required.

Now we may complete the proof of Proposition 6.4. Consider the following strategy st for Player I in the game $\partial_{\varepsilon, \kappa}^{c c}(\mathbb{Q})$. Suppose that the players arrived at stage $\alpha>0$ of the play and they have already constructed a sequence $\left\langle\bar{q}^{\beta}, \bar{p}^{\beta}, \varphi^{\beta}\right.$ : $\beta<\alpha\rangle$. Then, for each $j<\kappa^{+}$, the sequence $\left\langle p_{j}^{\beta}: \beta<\alpha\right\rangle$ is increasing, so Player I can take its least upper bound $q_{j}^{\alpha}$. This determines $\bar{q}^{\alpha}$ played by Player I; the function $\varphi^{\alpha}$ played at this stage is the $\varphi_{\bar{q}^{\alpha}}$ given by Claim 6.4.2.

One easily verifies that the strategy st is a winning one (remember Claim 6.4.1).

Theorem 6.5. Suppose $\theta$ and $\iota$ are cardinals such that $\theta=\operatorname{cf}(\theta)<\kappa \leq \iota=\iota^{<\kappa}$. Then the forcing notion $\mathbb{Q}$ is $(\theta, \iota, \kappa)$-manageable.

Proof. For each $\sigma \in \bigcup_{\alpha<\tau} \prod_{\beta<\alpha} \mu_{\beta}$ fix a sequence $\eta_{\sigma} \in \prod_{\alpha<\tau} \mu_{\alpha}$ such that $\sigma \subseteq \eta_{\sigma}$. Let $\bar{\eta}=\left\langle\eta_{\sigma}: \sigma \in \bigcup_{\alpha<\tau} \prod_{\beta<\alpha} \mu_{\beta}\right\rangle$.

Suppose that $N$ is a $(\mathbb{Q}, \kappa, \iota)$-relevant model such that $(\bar{\eta}, \bar{\mu}, \pi) \in N$.

For a condition $p \in \mathbb{Q}$ we define conditions $\mathrm{cl}_{N}^{+}(p)=q$ and $\mathrm{cl}_{N}^{-}(p)=r$ by

- $i^{r}=i^{q}=i^{p}$

- $u^{r}=\left(u^{p} \cap N\right) \cup\left\{\eta_{\sigma}: \sigma \in T\left(u^{p}\right) \cap N\right\}, u^{q}=u^{p} \cup\left\{\eta_{\sigma}: \sigma \in T\left(u^{p}\right) \cap N\right\}$,

- $f_{\sigma}^{r}=f_{\sigma}^{p}$ for $\sigma \in T\left(u^{p}\right) \cap N, f_{\sigma}^{q}=f_{\sigma}^{p}$ for $\sigma \in T\left(u^{p}\right)$, and $f_{\sigma}^{q}(j)=f_{\sigma}^{r}(j)=i^{p}$ for $\sigma \in T\left(u^{q}\right) \backslash T\left(u^{p}\right), j<i^{p}$

- $g^{r}(\eta)=g^{p}(\eta)$ for $\eta \in u^{p} \cap N$ and $g^{r}(\eta)=i^{p}$ for $\eta \in u^{r} \backslash u^{p}$; $g^{q}(\eta)=g^{p}(\eta)$ for $\eta \in u^{p}$ and $g^{q}(\eta)=i^{p}$ for $\eta \in u^{q} \backslash u^{p}$.

Plainly, $\operatorname{cl}_{N}^{+}(p), \operatorname{cl}_{N}^{-}(p)$ are conditions in $\mathbb{Q}$ and $\operatorname{cl}_{N}^{-}(p)$ belongs to $N$ (remember $<\kappa N \subseteq N)$. If $p \in N$, then also $\operatorname{cl}_{N}^{+}(p) \in N$.

Claim 6.5.1. Suppose that $p \in \mathbb{Q}, q \in N \cap \mathbb{Q}$ are such that $q \leq p$. Then

(1) $\operatorname{cl}_{N}^{+}(q)=\operatorname{cl}_{N}^{-}(q), q \leq \mathrm{cl}_{N}^{-}(p) \leq \mathrm{cl}_{N}^{+}(p)$, and $p \leq \mathrm{cl}_{N}^{+}(p)$,

(2) if $q^{\prime} \in N \cap \mathbb{Q}$ is stronger than $\mathrm{cl}_{N}^{-}(p)$, then $q^{\prime}$ and $p$ are compatible,

(3) if $p^{\prime} \in \mathbb{P}$ is stronger than $\operatorname{cl}_{N}^{+}(p)$, then $\operatorname{cl}_{N}^{-}(p) \leq \operatorname{cl}_{N}^{-}\left(p^{\prime}\right)$.

Proof of the Claim. (1) Just check.

(2) Suppose $\operatorname{cl}_{N}^{-}(p) \leq q^{\prime} \in N \cap \mathbb{Q}$. Put

$$
i^{r}=i^{q^{\prime}}, \quad u^{r}=u^{q^{\prime}} \cup u^{p}, \quad g^{r}(\eta)=\left\{\begin{array}{ll}
g^{q^{\prime}}(\eta) & \text { if } \eta \in u^{q^{\prime}}, \\
g^{p}(\eta) & \text { if } \eta \in u^{p} \backslash u^{q^{\prime}},
\end{array} \quad\right. \text { and: }
$$


if $\sigma \in T\left(u^{q^{\prime}}\right)$, then $f_{\sigma}^{r}=f_{\sigma}^{q^{\prime}}$, and if $\sigma \in T\left(u^{p}\right) \backslash T\left(u^{q^{\prime}}\right)$, then

$$
f_{\sigma}^{p} \subseteq f_{\sigma}^{r} \quad \text { and } \quad f_{\sigma}^{r}(j)=\sup \left\{\pi_{\eta}(j): \sigma \subseteq \eta \in u^{p}\right\}+1 \quad \text { for } i^{p} \leq j<i^{q^{\prime}} .
$$

Note that if $\eta \in u^{p} \backslash u^{q^{\prime}}$, then for some $\alpha<\tau$ we have $\eta\left\lceil\alpha \notin N\right.$ (so $\eta\left\lceil\alpha \notin T\left(u^{q^{\prime}}\right)\right.$ ). Hence we may easily verify that $r=\left(i^{r}, u^{r}, \bar{f}^{r}, g^{r}\right) \in \mathbb{Q}$ and clearly $r$ is stronger than $q^{\prime}$. To check that it is also stronger than $p$ it is enough to note that:

if $\eta \in u^{p} \cap u^{q^{\prime}}$, then $\left(\eta \in u^{\mathrm{cl}_{N}^{-}(p)}\right.$ and hence) $g^{q^{\prime}}(\eta)=g^{\mathrm{cl}_{N}^{-}(p)}(\eta)=g^{p}(\eta)$, and if $\sigma \in T\left(u^{p}\right) \cap T\left(u^{q^{\prime}}\right)$, then $\left(\sigma \in T\left(u^{\mathrm{cl}_{N}^{-}(p)}\right)\right.$ and hence) $f_{\sigma}^{p}=f_{\sigma}^{\mathrm{cl}_{N}^{-}(p)} \subseteq f_{\sigma}^{q^{\prime}}$.

(3) Note that if $\operatorname{cl}_{N}^{+}(p) \leq p^{\prime}$, then

$$
\left(u^{p} \cap N\right) \cup\left\{\eta_{\sigma}: \sigma \in T\left(u^{p}\right) \cap N\right\} \subseteq u^{p^{\prime}} \cap N,
$$

so checking the conditions for $\mathrm{cl}_{N}^{-}(p) \leq \mathrm{cl}_{N}^{-}\left(p^{\prime}\right)$ is pretty straightforward.

Claim 6.5.2. Suppose that a sequence $\left\langle p_{\zeta}: \zeta\left\langle\zeta^{*}\right\rangle \subseteq \mathbb{Q}\right.$ is increasing, $\zeta^{*}<\kappa$ is a limit ordinal, and $\mathrm{cl}_{N}^{+}\left(p_{\zeta}\right)=p_{\zeta+1}$ for all even $\zeta<\zeta^{*}$. Let $p^{*}$ be the least upper bound of $\left\langle p_{\zeta}: \zeta\left\langle\zeta^{*}\right\rangle\right.$. Then $\mathrm{cl}_{N}^{-}\left(p^{*}\right)$ is the least upper bound of $\left\langle\mathrm{cl}_{N}^{-}\left(p_{\zeta}\right): \zeta\left\langle\zeta^{*}\right\rangle\right.$.

Proof of the Claim. It follows from Claim 6.5.1(3) that $\mathrm{cl}_{N}^{-}\left(p_{\zeta}\right) \leq \mathrm{cl}_{N}^{-}\left(p^{*}\right)($ for $\zeta<$ $\left.\zeta^{*}\right)$. To show that $\operatorname{cl}_{N}^{-}\left(p^{*}\right)$ is actually the least upper bound it is enough to note that

$$
i^{\mathrm{cl}_{N}^{-}\left(p^{*}\right)}=i^{p^{*}}=\sup \left\{i^{p_{\zeta}}: \zeta<\zeta^{*}\right\}=\sup \left\{i^{\mathrm{cl}_{N}^{-}\left(p_{\zeta}\right)}: \zeta<\zeta^{*}\right\},
$$

and

$$
\begin{aligned}
& u^{p^{*}} \cap N=\bigcup\left\{u^{p_{\zeta+1}} \cap N: \zeta<\zeta^{*} \& \zeta \text { even }\right\}=\bigcup\left\{u^{\mathrm{cl}_{N}^{-}\left(p_{\zeta}\right)}: \zeta<\zeta^{*} \& \zeta \text { even }\right\}, \\
& \left\{\eta_{\sigma}: \sigma \in T\left(u^{p^{*}}\right) \cap N\right\}=\left\{\eta_{\sigma}: \sigma \in T\left(u^{p_{\zeta}}\right) \cap N \& \zeta<\zeta^{*}\right\} \subseteq \bigcup\left\{u^{\mathrm{cl}_{N}^{-}\left(p_{\zeta}\right)}: \zeta<\zeta^{*}\right\}
\end{aligned}
$$$$
\text { so } u^{\mathrm{cl}_{N}^{-}\left(p^{*}\right)}=u^{p^{*}} \cap N=\bigcup\left\{u^{\mathrm{cl}_{N}^{-}\left(p_{\zeta}\right)}: \zeta<\zeta^{*}\right\} \text {. }
$$

Now we may describe a strategy st for Her in the game $\partial^{m}(N, \theta, \mathbb{Q})$. Suppose that $i<\theta$ is even and $\left(q_{i}, p_{i}\right)$ is His move at this stage of the play (so $q_{i} \in N \cap \mathbb{P}$, $\left.q_{i} \leq p_{i} \in \mathbb{P}\right)$. Then st instructs Her to play $q_{i+1}=\operatorname{cl}_{N}^{-}\left(p_{i}\right)$ and $p_{i+1}=\operatorname{cl}_{N}^{+}\left(p_{i}\right)$. It follows from Claim 6.5.1(1) that $\left(q_{i+1}, p_{i+1}\right)$ is a legal move. It follows from Claims 6.5 .2 and 6.5.1 (2) that the strategy st is a winning one.

Thus we have shown that $\mathbb{Q}$ is weakly $(\theta, \iota, \kappa)$-manageable (remember Proposition 6.3(1)). The rest follows from Propositions 6.3 and 6.4.

Definition 6.6. We define $\mathbb{Q}$-names $\dot{f}_{\sigma}$ (for $\sigma \in \bigcup_{\alpha<\tau} \prod_{\beta<\alpha} \mu_{\beta}$ ) and $\dot{g}$ by

$$
\begin{array}{ll}
\Vdash_{\mathbb{Q}} & \text { " } \dot{f}_{\sigma}=\bigcup\left\{f_{\sigma}^{p}: p \in \Gamma_{\mathbb{Q}} \& \sigma \in T\left(u^{p}\right)\right\} ", \\
\Vdash_{\mathbb{Q}} & \text { " } \dot{g}=\bigcup\left\{g^{p}: p \in \Gamma_{\mathbb{Q}}\right\} " .
\end{array}
$$

Proposition 6.7. (1) $\Vdash_{\mathbb{Q}} " \dot{g}: \prod_{\alpha<\tau} \mu_{\alpha} \longrightarrow \kappa ”$.

(2) For each $\sigma \in \bigcup_{\alpha<\tau} \prod_{\beta<\alpha} \mu_{\beta}$ we have $\vdash_{\mathbb{Q}}$ " $\dot{f}_{\sigma}: \kappa \longrightarrow \kappa$ ”.

(3) For each $\eta \in \prod_{\alpha<\tau} \mu_{\alpha}$,

$$
\Vdash_{\mathbb{Q}} \cdots(\forall j<\kappa)\left(\dot{g}(\eta) \leq j \Rightarrow \pi_{\eta}(j)<\sup \left\{\dot{f}_{\eta\lceil\alpha}(j): \alpha<\tau\right\}\right) " .
$$


Proof. For $\eta \in \prod_{\alpha<\tau} \mu_{\alpha}$ and $i<\kappa$ let

$$
\mathcal{I}_{\eta}=\left\{p \in \mathbb{Q}: \eta \in u^{p}\right\} \quad \text { and } \quad \mathcal{I}^{i}=\left\{p \in \mathbb{Q}: i<i^{p}\right\} .
$$

We claim that these are open dense subsets of $\mathbb{Q}$. First, suppose $\eta \notin u^{p}, p \in \mathbb{Q}$ and let $i^{r}=i^{p}, u^{r}=u^{p} \cup\{\eta\}, g^{p} \subseteq g^{r}, g^{r}(\eta)=i^{r}, f_{\sigma}^{r}=f_{\sigma}^{p}$ for $\sigma \in T\left(u^{p}\right)$ and $f_{\eta\lceil\alpha}^{r}(j)=1$ if $\eta\left\lceil\alpha \notin T\left(u^{p}\right), \alpha<\tau\right.$. Then $r \in \mathcal{I}_{\eta}$ is stronger than $p$. (Thus the sets $\mathcal{I}_{\eta}$ are dense.)

Now suppose that $p \in \mathbb{P}$ is such that $i^{p} \leq i<\kappa$. Put $i^{r}=i+1, u^{r}=u^{p}, g^{r}=g^{p}$ and for $\sigma \in T\left(u^{r}\right)$ let $f_{\sigma}^{r} \supseteq f_{\sigma}^{p}$ be such that $\left(\operatorname{Dom}\left(f_{\sigma}^{r}\right)=i^{r}\right.$ and) for $j \in i^{r} \backslash i^{p}$ we have $f_{\sigma}^{r}(j)=\sup \left\{\pi_{\eta}(j): \eta \in u^{r}\right\}+1$. This way we have defined a condition $r \in \mathbb{Q}$ stronger than $p$ and such that $r \in \mathcal{I}^{i}$. (Thus the sets $\mathcal{I}^{i}$ are dense.)

Using the above observation and the definition of the order of $\mathbb{Q}$ one easily justifies (1) and (2). (Note also that, as $\mathbb{Q}$ is $<\kappa$-complete, $\Vdash_{\mathbb{Q}}$ " $\prod_{\alpha<\tau} \mu_{\alpha}=$ $\left(\prod_{\alpha<\tau} \mu_{\alpha}\right)^{\mathbf{V}}$ ".) Then (3) follows immediately once you note that

$$
p \Vdash " g^{p} \subseteq \dot{g} \& f_{\sigma}^{p} \subseteq \dot{f}_{\sigma} ",
$$

(for $\left.\sigma \in T\left(u^{p}\right), p \in \mathbb{Q}\right)$; remember Definition $6.2(1)(\mathrm{c})$.

\section{THE MODELS}

Theorem 7.1. Assume that

(a) $\kappa=2^{<\kappa}$,

(b) $\mu$ is a cardinal such that $\operatorname{cf}(\mu)<\kappa<\mu<\mu^{\operatorname{cf}(\mu)}=2^{\kappa}$,

(c) there is an increasing sequence $\bar{\mu}=\left\langle\mu_{\alpha}: \alpha<\operatorname{cf}(\mu)\right\rangle$ of regular cardinals such that

$(\forall \alpha<\operatorname{cf}(\mu))\left(\kappa \leq \mu_{\alpha} \leq\left(\mu_{\alpha}\right)^{\operatorname{cf}(\mu)} \leq \mu_{\alpha+1}\right) \quad$ and $\quad \mu=\sup \left\{\mu_{\alpha}: \alpha<\operatorname{cf}(\mu)\right\}$.

Then there is a forcing notion $\mathbb{P}$ such that:

(i) $\mathbb{P}$ has a dense subset of size $2^{\kappa}$,

(ii) $\mathbb{P}$ is $(\theta, \iota, \kappa)$-manageable for all cardinals $\theta, \iota$ satisfying $\operatorname{cf}(\theta)=\theta<\kappa \leq \iota=$ $\iota^{<\kappa}$,

(iii) $\Vdash_{\mathbb{P}}$ “ $\mathfrak{d}_{\kappa}^{\operatorname{cf}(\mu)} \leq \mu$ ",

(iv) if $\operatorname{cov}\left(\mathbf{M}_{\kappa, \kappa}\right)>\mu$, then $\Vdash_{\mathbb{P}}$ “ $\mathfrak{d}_{\kappa}^{\text {cf }(\mu)}=\mu<\left(\operatorname{cov}\left(\mathbf{M}_{\kappa, \kappa}\right)\right){ }^{\mathbf{V}} \leq \operatorname{cov}\left(\mathbf{M}_{\kappa, \kappa}\right)$ ".

Proof. Assume $\kappa, \mu, \bar{\mu}=\left\langle\mu_{\alpha}: \alpha<\operatorname{cf}(\mu)\right\rangle$ are as in the assumptions (a)-(c). Note that then also $\prod_{\alpha<\operatorname{cf}(\mu)} \mu_{\alpha}=2^{\kappa}$ (by Tarski's theorem).

The forcing notion $\mathbb{P}$ is built as the limit of a $<\kappa$-support iteration $\overline{\mathbb{Q}}=\left\langle\mathbb{P}_{\xi}, \dot{\mathbb{Q}}_{\xi}\right.$ : $\left.\xi<\kappa^{+}\right\rangle$. The names $\dot{\mathbb{Q}}_{\xi}$ are defined by induction on $\xi<\kappa^{+}$so that

( $\alpha) \mathbb{P}_{\xi}$ has a dense subset of size $2^{\kappa}$,

and for all cardinals $\theta, \iota$ satisfying $\operatorname{cf}(\theta)=\theta<\kappa \leq \iota=\iota^{<\kappa}$,

$(\beta)$ the forcing notion $\mathbb{P}_{\xi}$ is $(\theta, \iota, \kappa)$-manageable and

$(\gamma) \Vdash_{\mathbb{P}_{\xi}}$ " $\dot{\mathbb{Q}}_{\xi}$ is $(\theta, \iota, \kappa)$-manageable".

So suppose that $\mathbb{P}_{\xi}$ is already defined (and clauses $(\alpha),(\beta)$ hold). Then $\mathbb{P}_{\xi}$ is $<\kappa^{-}$ complete $\kappa^{+}-\mathrm{cc}$, and hence the properties of $\kappa, \mu$ and $\bar{\mu}$ stated in (a)-(c) hold in 
$\mathbf{V}^{\mathbb{P}_{\xi}}$. Take a $\mathbb{P}_{\xi}-$ name $\dot{\pi}_{\xi}$ such that

$$
\Vdash_{\mathbb{P}_{\xi}} \text { “ } \dot{\pi}_{\xi}: \prod_{\alpha<\operatorname{cf}(\mu)} \mu_{\alpha} \longrightarrow{ }^{\kappa} \kappa \text { is a bijection ", }
$$

and let $\dot{\mathbb{Q}}_{\xi}$ be a $\mathbb{P}_{\xi}$-name for the forcing notion $\mathbb{Q}\left(\dot{\pi}_{\xi}, \bar{\mu}, \kappa\right)$. Then clause $(\gamma)$ holds (remember Theorem 6.5).

It follows from Proposition 6.3(1) that the demand $(\alpha)$ is preserved at successor stages and it is preserved at limits $\leq \kappa^{+}$by the support we use. By Theorem 5.11. the clause $(\beta)$ holds for each $\mathbb{P}_{\xi}\left(\xi \leq \kappa^{+}\right)$. So our $\mathbb{P}=\mathbb{P}_{\kappa^{+}}$satisfies (i)+(ii).

For $\xi<\kappa^{+}$let $\dot{f}_{\sigma}^{\xi}$ (for $\sigma \in \bigcup_{\alpha<\operatorname{cf}(\mu)} \prod_{\beta<\alpha} \mu_{\beta}$ ) and $\dot{g}^{\xi}$ be $\mathbb{P}_{\xi+1}$-names for functions added by $\mathbb{Q}\left(\dot{\pi}_{\xi}, \bar{\mu}, \kappa\right)$ (see Definition 6.6). Then the family

$$
\mathcal{F}=\left\{\dot{f}_{\sigma}^{\xi}: \xi<\kappa^{+} \& \sigma \in \bigcup_{\alpha<\operatorname{cf}(\mu)} \prod_{\beta<\alpha} \mu_{\beta}\right\}
$$

is of size $\mu$. Since $\mathbb{P}$ is $\kappa^{+}$-cc, for each $\mathbb{P}$-name $\dot{h}$ for a member of ${ }^{\kappa} \kappa$, there are $\xi<\kappa^{+}$and a $\mathbb{P}_{\xi}$-name $\dot{h}^{*}$ such that $\Vdash_{\mathbb{P}} " \dot{h}=\dot{h}^{*}$ ". Thus using Proposition 6.7(3) we get

$\Vdash_{\mathbb{P}}$ " $\left(\exists j^{*}<\kappa\right)\left(\exists F \in \mathcal{P}_{\operatorname{cf}(\mu)^{+}}(\mathcal{F})\right)(\forall j<\kappa)\left(j^{*} \leq j \Rightarrow \dot{h}(j)<\sup \{f(j): f \in F\}\right)$ ".

Now we easily conclude that demand (iii) holds.

To show (iv) let us assume $\operatorname{cov}\left(\mathbf{M}_{\kappa, \kappa}\right)>\mu$. The forcing notion $\mathbb{P}$ is $\left(\aleph_{0}, \kappa, \kappa\right)-$ manageable, so by Corollary 5.10 and Proposition 4.5 we have

$$
\Vdash_{\mathbb{P}} " \mu<\left(\operatorname{cov}\left(\mathbf{M}_{\kappa, \kappa}\right)\right)^{\mathbf{V}} \leq \operatorname{cov}\left(\mathbf{M}_{\kappa, \kappa}\right) \leq \mathfrak{d}_{\kappa} " .
$$

By (iii) we know $\Vdash_{\mathbb{P}}$ " $\mathfrak{d}_{\kappa}^{\text {cf }(\mu)} \leq \mu$ ", but as for each $\alpha<\operatorname{cf}(\mu)$ :

$$
\Vdash_{\mathbb{P}} "\left(\mu_{\alpha}\right)^{\mathrm{cf}(\mu)} \leq \mu_{\alpha+1}<\mu<\mathfrak{d}_{\kappa} ",
$$

we immediately get that $\Vdash_{\mathbb{P}}$ " $\mathfrak{d}_{\kappa}^{\operatorname{cf}(\mu)}=\mu$ " (remember $\left.\mathfrak{d}_{\kappa} \leq\left(\mathfrak{d}_{\kappa}^{\operatorname{cf}(\mu)}\right)^{\operatorname{cf}(\mu)}\right)$.

Corollary 7.2. Assume GCH. Then there a $\kappa$-complete $\kappa^{+}-c c$ forcing notion $\mathbb{P}^{*}$ such that

$$
\Vdash_{\mathbb{P}^{*}} \text { “ } \overline{\mathfrak{d}}_{\kappa}=\mathfrak{d}_{\kappa}^{\aleph_{0}}=\kappa^{+\omega} \quad \text { and } \operatorname{cov}\left(\mathbf{M}_{\kappa, \kappa}\right)=2^{\kappa}=\kappa^{+(\omega+1)} " .
$$

Proof. Let $\mathbb{P}_{0}=\mathbb{C}_{\kappa^{+(\omega+1)}, \kappa}$ be the forcing adding $\kappa^{+(\omega+1)}$ many Cohen functions in $\kappa_{\kappa}$ (with $<\kappa$-support). Note that

$$
\Vdash_{\mathbb{P}_{0}} \text { “ } \kappa, \mu=\kappa^{+\omega} \text { and } \bar{\mu}=\left\langle\kappa^{+n}: n<\omega\right\rangle \text { are as in Theorem 7.1(a)-(c)". }
$$

Therefore we have a $\mathbb{P}_{0}$-name $\dot{\mathbb{P}}$ for a forcing notion satisfying 7.1(i)-(iv). (Note that $\Vdash_{\mathbb{P}_{0}}$ " $\operatorname{cov}\left(\mathbf{M}_{\kappa, \kappa}\right)=\kappa^{+(\omega+1)}$ ", so the assumption of 7.1 (iv) holds.) Let $\mathbb{P}^{*}=$ $\mathbb{P}_{0} * \dot{\mathbb{P}}$.

Note that Theorem 0.1 follows from Corollary [7.2, Theorem 1.12, and the fact that $\operatorname{cov}\left(\mathbf{M}_{\kappa, \kappa}\right) \leq \operatorname{cof}\left(\mathcal{N} S_{\kappa}\right)$.

Theorem 7.3. Assume that (a)-(c) of Theorem 7.1 hold and

(d) $\nu$ is a regular cardinal such that $\mu<\nu<2^{\kappa}$.

Then there is a forcing notion $\mathbb{P}_{\nu}$ satisfying (i)+(ii) of Theorem 7.1$]$ and

(iii) ${ }_{\nu}^{+} \Vdash_{\mathbb{P}_{\nu}}$ “ $\mathfrak{d}_{\kappa}^{\tau}=\nu$ for every cardinal $\tau$ satisfying $\operatorname{cf}(\mu) \leq \tau<\kappa$ ”,

(iv) ${ }^{-} \Vdash_{\mathbb{P}_{\nu}} "\left(\operatorname{cov}\left(\mathbf{M}_{\kappa, \kappa}\right)\right)^{\mathbf{V}} \leq \operatorname{cov}\left(\mathbf{M}_{\kappa, \kappa}\right) "$. 
Proof. The forcing notion $\mathbb{P}_{\nu}$ is the limit of $<\kappa$-support iteration $\overline{\mathbb{Q}}=\left\langle\mathbb{P}_{\xi}, \dot{\mathbb{Q}}_{\xi}: \xi<\right.$ $\nu\rangle$, where $\dot{\mathbb{Q}}_{\xi}$ are defined as in the proof of Theorem[7.1 (so the only difference is the length of the iteration). As there, $\mathbb{P}_{\nu}$ satisfies (i)+(ii) and $\Vdash_{\mathbb{P}_{\nu}}$ " $\mathfrak{d}_{\kappa}^{\operatorname{cf}(\mu)} \leq \nu$ ". Since $\operatorname{cov}\left(\mathbf{M}_{\kappa, \kappa}\right)>\kappa$ and $\mathbb{P}_{\nu}$ is $\left(\aleph_{0}, \kappa, \kappa\right)$-manageable, we get (iv) ${ }^{-}$(by Corollary [5.10). To show that (iii) ${ }_{\nu}^{+}$holds, suppose that $\dot{\mathcal{F}}$ is a $\mathbb{P}_{\nu}-$ name for a family of functions in ${ }^{\kappa} \kappa$ of size $<\nu$. Then $\dot{\mathcal{F}}$ is essentially a $\mathbb{P}_{\xi}$-name for some $\xi<\nu$. Since $\mathbb{P}_{\xi+\kappa}$ adds a subset of $\kappa$ which is Cohen over $\mathbf{V}^{\mathbb{P}_{\xi}},\left({ }^{\kappa} \kappa\right)^{\mathbf{V}^{\mathbb{P}}}$ is not a dominating family in $\left(\kappa_{\kappa}\right)^{\mathbf{V}^{\mathbb{P}_{\nu}}}$, and hence for any $\tau<\kappa$,

$$
\Vdash_{\mathbb{P}_{\nu}} \text { “ } \dot{\mathcal{F}} \text { is not } \tau \text {-dominating } "
$$

(remember that $\mathbb{P}_{\nu}$ is $<\kappa-$-complete).

Now, Theorem 0.2 follows from Theorem 1.12 and the following corollary.

Corollary 7.4. It is consistent, relative to the existence of a cardinal $\nu$ such that $o(\nu)=\nu^{++}$, that $\overline{\mathfrak{d}}_{\aleph_{1}}=\aleph_{\omega+1}$ and $\operatorname{cov}\left(\mathbf{M}_{\aleph_{1}, \aleph_{1}}\right)=\aleph_{\omega+2}$.

Proof. Gitik and Woodin (see Gitik [4] also Gitik and Merimovich [5]) constructed a model of " $2^{\aleph_{n}}<\aleph_{\omega}$ for every $n<\omega, 2^{\aleph_{0}}=\aleph_{1}$ and $2^{\aleph_{\omega}}=\aleph_{\omega+2}$ " from $o(\nu)=\nu^{++}$. Add $\aleph_{\omega+2}$ Cohen subsets of $\aleph_{1}$ (with countable support) to that model and then apply Theorem 7.3 (with $\kappa=\aleph_{1}, \mu=\aleph_{\omega}$ and $\left.\nu=\aleph_{\omega+1}\right)$.

Theorem 7.5. Assume that

(a) $\kappa=\operatorname{cf}(\kappa)=2^{<\kappa}, n<\omega$,

(b) $\mu_{0}, \mu_{1}, \ldots, \mu_{n}$ are cardinals such that

$$
\mu_{0}>\mu_{1}>\ldots>\mu_{n}>\kappa \text { and } \operatorname{cf}\left(\mu_{0}\right)<\operatorname{cf}\left(\mu_{1}\right)<\ldots<\operatorname{cf}\left(\mu_{n}\right)<\kappa,
$$

(c) $\left(\mu_{\ell}\right)^{\mathrm{cf}\left(\mu_{\ell}\right)}=2^{\kappa}$ for $\ell=0, \ldots, n$,

(d) for $\ell=0, \ldots, n$, there is an increasing sequence $\bar{\mu}^{\ell}=\left\langle\mu_{\alpha}^{\ell}: \alpha<\operatorname{cf}\left(\mu_{\ell}\right)\right\rangle$ of regular cardinals such that

$$
\left(\forall \alpha<\operatorname{cf}\left(\mu_{\ell}\right)\right)\left(\kappa \leq \mu_{\alpha}^{\ell}=\left(\mu_{\alpha}^{\ell}\right)^{\operatorname{cf}\left(\mu_{\ell}\right)}\right) \quad \text { and } \quad \mu_{\ell}=\sup \left\{\mu_{\alpha}^{\ell}: \alpha<\operatorname{cf}\left(\mu_{\ell}\right)\right\},
$$

(e) $\operatorname{cov}\left(\mathbf{M}_{\kappa, \kappa}\right)=2^{\kappa}$.

Then there is a forcing notion $\mathbb{P}$ such that

(i) $\mathbb{P}$ has a dense subset of size $2^{\kappa}$,

(ii) $\mathbb{P}$ is $(\theta, \iota, \kappa)$-manageable for all cardinals $\theta, \iota$ satisfying $\operatorname{cf}(\theta)=\theta<\kappa \leq \iota=$ $\iota^{<\kappa}$,

(iii) $\Vdash_{\mathbb{P}}$ “ $\mathfrak{d}_{\kappa}^{\text {cf( }\left(\mu_{\ell}\right)}=\mu_{\ell}$ for $\ell=0, \ldots, n$ and $\operatorname{cov}\left(\mathbf{M}_{\kappa, \kappa}\right)=2^{\kappa} "$

Proof. Let $A_{0}, \ldots, A_{n}$ be a partition of $\kappa^{+}$into sets of size $\kappa^{+}$. The forcing notion $\mathbb{P}$ is the limit of a $<\kappa$-support iteration $\overline{\mathbb{Q}}=\left\langle\mathbb{P}_{\xi}, \dot{\mathbb{Q}}_{\xi}: \xi<\kappa^{+}\right\rangle$defined as in the proof of Theorem 7.1, but

- if $\xi \in A_{\ell}$, then $\dot{\pi}_{\xi}$ is a $\mathbb{P}_{\xi}$-name for a bijection from $\prod_{\alpha<\tau_{\ell}} \mu_{\alpha}^{\ell}$ onto ${ }^{\kappa} \kappa$, and $\dot{\mathbb{Q}}_{\xi}$ is $\mathbb{Q}\left(\dot{\pi}_{\xi}, \bar{\mu}^{\ell}, \kappa\right)$.

We argue that $\mathbb{P}$ has the required properties similarly as in Theorem 7.1 
Remark 7.6. Of course the assumption (e) in Theorem 7.5 is not very important: we may start with adding $2^{\kappa}$ many Cohen subsets of $\kappa$.

Corollary 7.7. It is consistent, relative to the existence of a strong cardinal, that

$$
\mathfrak{d}_{\aleph_{2}}^{\aleph_{1}}=\aleph_{\omega_{1}}, \quad \mathfrak{d}_{\aleph_{2}}^{\aleph_{0}}=\aleph_{\omega_{1}+\omega} \quad \text { and } \quad \operatorname{cov}\left(\mathbf{M}_{\aleph_{2}, \aleph_{2}}\right)=\aleph_{\omega_{1}+\omega+1}
$$

Proof. As was pointed out to us by Moti Gitik, by work of Magidor [8], Merimovich [12, and Segal [13], it is consistent relative to a strong cardinal that

(a) $2^{\aleph_{1}}=\aleph_{2}, \aleph_{\omega_{1}}^{\aleph_{0}}=\aleph_{\omega_{1}}$, and

(b) for every $\alpha<\omega_{1}$,

$$
\aleph_{\alpha+\omega+1}^{\aleph_{1}}=\aleph_{\alpha+\omega+1} \quad \text { and } \quad 2^{\aleph_{\alpha+\omega+1}}=\aleph_{\alpha+\omega+2},
$$

and

(c) $\aleph_{\omega_{1}}^{\aleph_{1}}=\aleph_{\omega_{1}+\omega}^{\aleph_{0}}=\aleph_{\omega_{1}+\omega+1}$.

After adding $\aleph_{\omega_{1}+\omega+1}$ Cohen subsets of $\aleph_{2}$ (with $<\aleph_{2}$ support) to a model of (a)(c) we will get a model in which the assumptions of Theorem 7.5 are satisfied for $\kappa=\aleph_{2}, n=2, \mu_{0}=\aleph_{\omega_{1}+\omega}, \mu_{1}=\aleph_{\omega_{1}}$.

\section{REFERENCES}

[1] Yoshihiro Abe. A hierarchy of filters smaller than $\mathrm{CF}_{\kappa \lambda}$. Archive for Mathematical Logic, 36:385-397, 1997. MR:1477763 (98m:03098)

[2] Bohuslav Balcar and Petr Simon. Disjoint refinement. In Handbook of Boolean algebras, volume 2, pages 333-388. North-Holland, Amsterdam, 1989. MR0991597

[3] James Cummings and Saharon Shelah. Cardinal invariants above the continuum. Annals of Pure and Applied Logic, 75:251-268, 1995. math.LO/9509228. MR1355135 (96k:03117)

[4] Moti Gitik. The negation of the singular cardinal hypothesis from $o(\kappa)=\kappa^{++}$. Annals of Pure and Applied Logic, 43:209-234, 1989. MR1007865 (90h:03037)

[5] Moti Gitik and Carmi Merimovich. Possible values for $2^{\aleph_{n}}$ and $2^{\aleph_{\omega}}$. Annals of Pure and Applied Logic, 90:193-241, 1997. MR 1489309 (2000a:03084)

[6] Thomas Jech. Set theory. Academic Press, New York, 1978. MR0506523 (80a:03062)

[7] Avner Landver. Singular Baire numbers and related topics. Ph.D. thesis, University of Wisconsin, Madison, 1990.

[8] Menachem Magidor. On the singular cardinals problem I. Israel J. Math., 28:1-31, 1977. MR0491183 (58:10449a)

[9] Pierre Matet and Janusz Pawlikowski. $Q$-pointness, $P$-pointness and feebleness of ideals. Journal of Symbolic Logic, 68:235-261, 2003. MR.1959318 (2004c:03057)

[10] Pierre Matet, Cédric Péan, and Saharon Shelah. Cofinality of normal ideals on $P_{\kappa}(\lambda)$, I. Archive for Mathematical Logic. math.LO/0404318.

[11] Pierre Matet, Cédric Péan, and Saharon Shelah. Cofinality of normal ideals on $P_{\kappa}(\lambda)$, II. Israel Journal of Mathematics, to appear.

[12] Carmi Merimovich. Extender-based Radin forcing. Transactions of the American Mathematical Society, 355:1729-1772, 2003. MR1953523 (2004d:03108)

[13] Miri Segal. Master Thesis. The Hebrew University of Jerusalem, 1996. Menachem Magidor, adviser.

[14] Saharon Shelah. A weak generalization of MA to higher cardinals. Israel Journal of Mathematics, 30:297-306, 1978. MR0505492 (58:21606)

[15] Saharon Shelah. Strong Partition Relations Below the Power Set: Consistency, Was Sierpiński Right, II? In Proceedings of the Conference on Set Theory and its Applications in honor of A.Hajnal and V.T.Sos, Budapest, 1/91, volume 60 of Colloquia Mathematica Societatis Janos Bolyai. Sets, Graphs, and Numbers, pages 637-638. 1991. math.LO/9201244. MR1218224 (95b:03052)

[16] Saharon Shelah. Was Sierpiński right? IV. Journal of Symbolic Logic, 65:1031-1054, 2000. math.LO/9712282. MR1791363 (2001k:03092) 
[17] Saharon Shelah and Lee Stanley. Generalized Martin's axiom and Souslin's hypothesis for higher cardinals. Israel Journal of Mathematics, 43:225-236, 1982. Corrections in [Sh:154a].

[18] Saharon Shelah and Lee Stanley. Corrigendum to: "Generalized Martin's axiom and Souslin's hypothesis for higher cardinals" [Israel Journal of Mathematics 43 (1982), no. 3, 225-236; MR 84h:03120]. Israel Journal of Mathematics, 53:304-314, 1986.

Departement de Mathématiques, Université de Caen - CNRS, BP 5186, 14032 Caen Cedex, France

E-mail address: matet@math.unicaen.fr

Department of Mathematics, University of Nebraska at Omaha, Omaha, Nebraska $68182-0243$

E-mail address: roslanow@member.ams.org

URL: http://www . unomaha.edu/logic

Institute of Mathematics, The Hebrew University of Jerusalem, 91904 Jerusalem, Israel - And - Department of Mathematics, Rutgers University, New Brunswick, New JERSEY 08854

E-mail address: shelah@math.huji.ac.il

$U R L:$ http://shelah.logic.at 